\title{
The interaction of Blasius boundary-layer flow with a compliant panel: Global, local and transient analyses
}

\footnotetext{
${ }^{1}$ Fluid Dynamics Research Group, Department of Mechanical Engineering, Curtin University,
} Western Australia 6845, Australia

(Received xx; revised xx; accepted xx)

We study the fluid-structure interaction (FSI) of a compliant panel with developing Blasius boundary-layer flow. The linearised Navier-Stokes equations in velocity-vorticity form are solved using a Helmholtz decomposition coupled with the dynamics of a platespring compliant panel couched in finite-difference form. The FSI system is written as an eigenvalue problem and the various flow- and wall-based instabilities are analysed. It is shown that global temporal instability can occur through the interaction of TravellingWave Flutter (TWF) with a structural mode or as a resonance between TollmienSchlichting Wave (TSW) instability and discrete structural modes of the compliant panel. The former is independent of compliant-panel length and upstream inflow disturbances while the specific behaviour arising from the latter phenomenon is dependent upon the frequency of a disturbance introduced upstream of the compliant panel. The inclusion of axial displacements in the wall model does not lead to any further global instabilities. The dependence of instability-onset Reynolds numbers with structural stiffness and damping for the global modes is quantified. It is also shown that the TWF-based global instability is stabilised as the boundary layer progresses downstream while the TSW-based global instability exhibits discrete resonance-type behaviour with increases to Reynolds number. At sufficiently high Reynolds numbers, a globally unstable divergence instability is identified when the wavelength of its wall-based mode is longer than that of the least stable TSW mode. Finally, a non-modal analysis reveals a high level of transient growth when the flow interacts with a compliant panel which has structural properties capable of reducing TSW growth but which is prone to global instability through wall-based modes.

\section{Introduction}

This paper is motivated by the potential of compliant panels to interact favourably with the dynamics of boundary-layer flows and thereby yield a reduction to skin-friction drag. The investigation focuses upon instability mechanisms that can lead to laminarto-turbulent transition in low-disturbance environments for zero pressure-gradient boundary-layer flow although the new modelling approach developed is readily extended to the study of other wall-bounded mean shear flows with a deformable boundary.

There exists a rich literature on the topic of 'compliant walls for transition delay' spanning the nearly sixty years since the pioneering experimental work of Kramer (1957, 1960); this has been summarised periodically through review articles (Benjamin 1963; Bushnell 1977; Carpenter 1991; Gad-el-Hak 1998; Carpenter et al. 2001) that chronicle the development of the field of study. The principal engineering outcome to date is that 


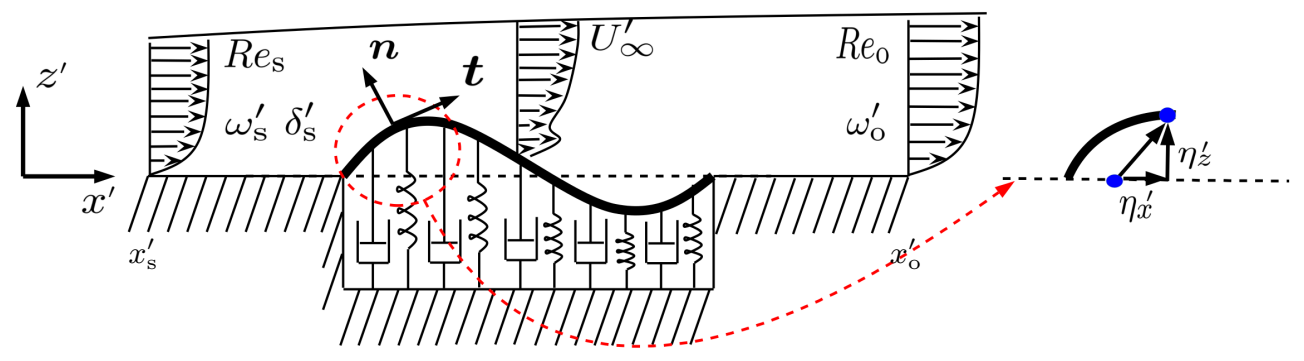

Figure 1: Schematic of the system studied with nomenclature.

optimally-designed compliant coatings could extend the length of the linear transition regime by a factor of 5.7 (Dixon et al. 1994) and the prediction, argued in Carpenter et al. (2001), that a series of compliant panels, each with properties tailored to local mean flow, could theoretically postpone transition indefinitely. In contrast, a focus over the last decade or so on establishing a theoretical framework for compliant-wall interactions with turbulent boundary layers (Rempfer et al. 2001; Xu et al. 2003; Fukagata et al. 2008; Kim \& Choi 2014; Luhar et al. 2015, 2016) to understand, inter alia, the experimentally measured drag-reductions in Choi et al. (1997) at present suggests that only limited benefits are conferred by wall compliance. Competing passive technologies such as the use of riblets currently offer a more practicable strategy for drag-reduction in turbulent boundary-layer flow. Nevertheless, the technological prospect of turbulent skin-friction drag reduction using wall compliance remains an open question. However, the present study returns to the theme of transition postponement and serves to yield a complete understanding of the interaction of laminar boundary-layer flow with compliant panels, i.e. compliant coatings of finite streamwise extent. A schematic of the system studied is presented in Figure 1.

Hitherto, theoretical studies of laminar boundary-layer flow over a compliant wall have been undertaken mainly using two-dimensional (2D) (Carpenter \& Garrad 1985, 1986; Sen \& Arora 1988) or three-dimensional (3D) (Yeo 1992; Joslin et al. 1991; Joslin \& Morris 1992; Zengl \& Rist 2012) linear temporal or spatial local stability analyses based on the assumption of a compliant wall of infinite extent. Numerical simulations of compliant panels interacting with laminar shear flow have been performed for linear (Davies \& Carpenter 1997b) and nonlinear (Wiplier \& Ehrenstein 2000, 2001; Pavlov 2006) system perturbations but these have been limited by the choice of type and frequency of the initial disturbance and therefore their results pertain to a limited domain within the overall stability space. Emphasis has been placed on the effect of surface-based or volumebased isotropic and anisotropic compliant walls (Zengl \& Rist 2012; Pavlov 2006; Yeo \& Dowling 1987; Yeo 1988, 1990; Carpenter \& Morris 1990) on both 2D and 3D instabilities in order to identify rationally coating parameters (Dixon et al. 1994; Carpenter 1993) that are suitable for postponing the onset of spatially-growing instabilities to higher Reynolds numbers (hence distance downstream of the leading edge in applications) or reducing their amplification. Using the aforementioned techniques, three types of instability have been identified and categorised as: (i) flow-based, (ii) flow-induced wall-based instabilities, and (iii) instabilities arising from linear modal interactions. These types of instability are each expanded upon in the following three paragraphs.

Amplifying Tollmien-Schlichting Waves (TSWs) (Schlichting 1979) comprise the main flow-based instability; these are also responsible for natural transition to turbulence in flat-plate boundary-layer flow over rigid walls in low-disturbance environments. However, 
in a convective 2D boundary-layer flow, an additional flow-based transient mechanism exists, namely the Orr instability (Orr 1907; Butler \& Farrell 1992; Åkervik et al. 2007), which extracts energy from the mean shear by transporting momentum downstream through the action of the perturbation Reynolds stress. Disturbances, which are tilted against the shear first rise to an upright position while drawing energy from the mean flow after which energy is returned to the mean flow further downstream. This energytransfer mechanism can potentially precipitate transition to turbulence, by-passing the TSW route in natural transition.

The flow-induced wall-based instabilities principally comprise travelling-wave flutter (TWF) (Carpenter \& Garrad 1986; Yeo 1988) and static divergence (SD). TWF is the destabilisation of free surface wave modes of the compliant wall through the fluid loading and takes the form of a wave propagating downstream with a phase speed close to that of the free-stream flow. In contrast, static divergence (SD) (Lucey \& Carpenter 1992; Pitman \& Lucey 2009) occurs when the hydrodynamic forces generated by a wall deformation exceed the associated restorative forces in the compliant wall and manifests itself as a slow downstream travelling wave. Like TSWs, TWF is a convective instability with a wave amplifying only as it propagates away from the initiating source of disturbance, while SD is considered an absolute instability because the wave grows in time at all spatial locations of the domain. Even though the mechanism underlying TWF is essentially inviscid, the Orr-Sommerfeld equation can accurately predict its onset Reynolds number and subsequent propagation. However, it largely fails to predict the absolute SD (Carpenter \& Morris 1990) because exactly at onset the instability is static and therefore the wave-based assumption of the Orr-Sommerfeld equation is essentially invalid. A further flow-induced surface instability, associated with the tangential (axial) wall motion, was discovered by Shankar \& Kumaran (2002) when studying the local temporal stability and asymptotic analysis of Couette flow past a flexible surface. This instability occurs through energy transfer to the wall caused by the interaction of the fluctuating (fluid) shear stress and the axial motion of the wall at the fluid-solid interface. However, less is known about the character of this new instability - whether it is convective or absolute - and its interaction with structural modes or the TSW or TWF instabilities in a FSI system of finite length.

The third category of instability includes transitional instability (Sen \& Arora 1988) which is generated by the coalescence of TWF and TSWs and the instability caused by the coalescence of the evanescent waves and a TSW (Wiplier \& Ehrenstein 2001). These instabilities are identified in a local stability analysis as a pinching of the different instability branches in the wavenumber $(\alpha)$ plane for positive imaginary part of the frequency $(\omega)$ or, equivalently, as a cusp in the frequency plane (Wiplier \& Ehrenstein 2001; Yeo et al. 1996) at the frequency where the coalescence of the two modal branches takes place. These are both absolute instabilities and, along with SD, must be avoided in compliant-wall design because their occurrence is likely to result in a significant modification to the base flow (Wiplier \& Ehrenstein 2001; Carpenter \& Morris 1990) and/or premature boundary-layer transition.

In the energy classification of Benjamin (1963), TSWs are found to be a Class A instability because their activation energy (energy relative to the quiescent system state) is negative and they are therefore destabilized by structural damping since it removes energy from the FSI system. These waves are equivalently termed Negative-Energy Waves (NEWs) in, for example, (Crighton \& Oswell 1991), this description having been adopted from the plasma-physics community. TWF is a Class B instability because its activation energy is positive (hence a Positive-Energy Wave in Crighton \& Oswell (1991)) and is therefore attenuated by the action of structural damping. Finally, Class $\mathrm{C}$ comprises 
waves that are destabilized independently of whether there is irreversible energy transfer from/to the FSI system. Kelvin-Helmholtz instability (called modal-coalescence flutter in the hydro-elasticity of flexible panels and walls) and the transitional instability belong to this class of instability. The SD waves have been predicted to be Class A when modelled using an infinitely long domain (e.g. Crighton \& Oswell (1991), Davies \& Carpenter $(1997 a)$ ) because structural damping is required to precipitate the instability. However, for compliant panels of finite length it has been shown (Lucey \& Carpenter 1992; Pitman \& Lucey 2009) that structural damping reverts to its conventional rôle and reduces the amplification of SD waves at post-critical flow speeds. In addition, its mechanism is principally due to conservative energy exchanges thereby suggesting that it is more likely to be a Class C instability.

Although local stability analyses have revealed the main physics behind the rich range of phenomena supported by the FSI system, there remain drawbacks arising from the local nature of the analysis. Specifically, it is difficult to model the boundary conditions of a compliant wall of finite extent because the analysis assumes homogeneity in the streamwise direction. The non-trivial effect on the system's stability due to panel edges which can reflect incident waves has been emphasized by several investigations both in the potential (Lucey \& Carpenter 1992; Pitman \& Lucey 2009; Peake 2004) and viscous (Davies \& Carpenter 1997 b; Wiplier \& Ehrenstein 2001; Stewart et al. 2009) flow regimes. For potential flow, Peake (2004) showed that a long but finite plate possesses resonant solutions and that the temporal instability is present in the absence of structural damping on the finite plate even for cases where a local analysis predicts that the flow is stable. This finding agreed with the theoretical ideas of Lucey \& Carpenter (1993) and were confirmed by numerical simulations of the system by Lucey \& Carpenter (1992). Stewart et al. (2009) demonstrated that a local analysis gives only limited insight into the properties of the global system because wave reflections at boundaries played a vital role in the growth of mode- 1 oscillations in their finite-length compliant insert in the wall of a two-dimensional channel. However, it is not known whether higher-order eigenmodes of the finite structure can interact with modes of the viscous flow and whether this interaction might be identified in a local stability analysis. In addition, to study absolute instabilities that may exist in the system, the frequency must be varied in order to reveal the entire unstable wavenumber spectrum, $\alpha(\omega)$, and identify the different instability branches for a specific Reynolds number and their possible pinching; this proves to be a daunting task (Wiplier \& Ehrenstein 2001; Yeo et al. 1996). In parallel, it has been shown through a multiple-scale analysis (Yeo et al. 1994) that the incorporation of non-parallel effects due to boundary-layer growth (clearly omitted in a local analysis) are destabilising for TSW and TWF instabilities for Blasius flow over a compliant wall. The modelling approach, built on global stability considerations, developed and deployed in the present paper serves to overcome the limitations of local analyses.

We acknowledge that the stability of finite-length flexible plates subjected to boundarylayer flow at transonic and low subsonic flow speeds, for which plate flutter is usually the critical instability has been well studied. Dowell $(1971,1973)$ modelled the effects of a laminar boundary layer analytically while more recent work by Hashimoto et al. (2009) and Alder $(2015,2016)$ incorporated the effects of turbulent boundary layers by solving the Reynolds-averaged Navier Stokes equations. The overall findings of these studies are that the boundary-layer exercises a stabilising effect, as compared with potential flow predictions, on panel flutter at low supersonic Mach numbers. However, the present work represents the first fairly complete stability analysis of boundary-layer flow over a finite compliant panel at low, incompressible, flow speeds.

Advances in algorithms for the numerical solution of large nonsymmetric complex gen- 
eralized eigenvalue problems have resulted in the ability to extend local stability theory to two inhomogeneous directions with one homogeneous direction and this defines the term 'bi-global' stability. In this approach, the time asymptotic and transient behaviour for a wide variety of 2D and 3D flows has become attainable (Theofilis 2003, 2011). Based on the analysis of Cossu \& Chomaz (1997) and Chomaz (2005), Ehrenstein \& Gallaire (2005) investigated the convective mechanism of the classical flat-plate boundary layer by means of an appropriate superposition of two-dimensional global modes. They found that a superposition of the damped temporal global TSW eigenmodes gave rise to a localized wavepacket at the inflow boundary. The wavepacket would then grow while being advected downstream, in close agreement with direct numerical simulation results. However, the transient growth in energy was only of one order in magnitude. However, Åkervik et al. (2007), who identified all the modal branches, found that for a slightly non-parallel flow, many eigenmodes are needed in order to obtain a converged transient energy result and that it is the combination the Orr and TSW mechanisms which yielded the potential for large downstream amplification through transient growth.

The first study of the global stability of $2 \mathrm{D}$ disturbances in plane Poiseuille flow over a compliant wall with periodic boundary conditions was conducted by Pitman \& Lucey (2010), using a velocity-vorticity formulation of the Navier-Stokes equations (Davies \& Carpenter 2001) combined with the vortex-source sheet boundary-integral method. They found that a flexible wall with structural damping improved the global system's temporal stability by $4 \%$ at $R e=6000$ and that a compliant wall with the properties of Davies \& Carpenter (1997b) results in a significant deterioration of system stability.

The present work extends the velocity-vorticity formulation combined with the generalized Helmholtz decomposition (Wu \& Thompson 1973; Kempka et al. 1995) to the global stability of the Blasius flow over a compliant wall taking into account both vertical and axial structural displacements. This is used to investigate the asymptotic and transient behaviour of the FSI system to 2D incident disturbances. Local spatial stability analysis is also conducted in order to validate the global stability model but also to reveal the spatial characteristics of the predicted temporal instabilities. Inhomogeneity in the streamwise direction due to the boundary conditions of the compliant panel and boundary-layer growth are incorporated in the model. All of the flow and structural eigenfrequencies and the different instability branches are obtained through the solution of a generalized eigenvalue problem. In this way, it is possible to investigate the interaction between modes and identify the conditions for temporal instability in the FSI system.

In addition, the evaluation of all eigenmodes in the present study permits the transient response of the FSI system to incident 2D disturbances to be studied. This reveals the potential of the compliant panel either to damp out disturbances in the convectively unstable boundary-layer flow or to amplify them by transferring a significant amount of energy as they travel downstream, thereby enhancing the conditions for by-pass transition. A similar investigation was conducted by Zengl \& Rist (2012) for isotropic and anisotropic compliant-wall materials for 3D disturbances via a local stability analysis. They found that in addition to the main mechanism for the transient growth of disturbances over a rigid wall, another oscillatory mechanism coexists in the presence of an anisotropic compliant wall but that the maximum transient growth and the time of its occurrence are hardly affected.

This paper is laid out as follows. The problem formulation is presented in Section 2, where the equations for the mean Blasius flow, the $2 \mathrm{D}$ flow field for the disturbances, the Helmholtz decomposition and the linear model of the compliant wall's structural dynamics are developed for both global and local stability analyses. Thereafter, the equations that describe the linear global transient response of the FSI system to 2D 
disturbances, are presented and finally the discretisation of the equations using the boundary-integral vortex-source sheet method are presented. In Section 3 the choice of parameters is justified and results from global and local stability investigations are presented and discussed. Particular focus is placed upon interactions between the different types of flow- and wall-based modes for a compliant-wall model that permits only vertical structural displacements. The effect of both vertical and axial structural displacements is then considered as a modification to the main stability results. The global transient response of the FSI system to 2D incident disturbances is then presented. In Section 4 we provide a summary of the main findings of the investigation and draw conclusions. Finally, we remark that the theoretical approach and the discovery of two types of global instability were presented orally at an IUTAM symposium in 2014 with a summary published (Tsigklifis \& Lucey 2015) in a special issue that recorded the outcomes of the symposium. The present paper provides a complete description of the theoretical methods with a full investigation, including parametric dependence, of globally unstable modes, their relationships to local modes, and transient effects in boundary-layer flow over a compliant panel.

\section{Problem formulation}

As illustrated in figure 1, a Blasius boundary layer progresses over a rigid-wall section of length $L_{\mathrm{w} 1}^{\prime}$ onto a compliant panel of length $L_{\mathrm{c}}^{\prime}$ comprising a spring-backed flexible plate (that may include a dashpot-type damping) with which it interacts, and finally over a rigid-wall section of length $L_{\mathrm{w} 2}^{\prime}$. Here and hereafter, ' denotes a dimensional quantity. At entry and exit (respectively distances $x_{\mathrm{s}}^{\prime}$ and $x_{\mathrm{o}}^{\prime}$ downstream from the origin of the growing boundary layer) to the domain the Reynolds number (based upon free-stream flow speed $U_{\infty}^{\prime}$, fluid density $\rho_{1}^{\prime}$ and dynamic viscosity $\mu_{1}^{\prime}$, and boundarylayer displacement thickness $\delta^{\prime}$ ) are respectively $R e_{\mathrm{s}}$ and $R e_{\mathrm{o}} ; \omega_{\mathrm{s}}^{\prime}$ and $\omega_{\mathrm{o}}^{\prime}$ are the radian frequencies of perturbation waves that satisfy the Orr-Sommerfeld equation that serve as entry and exit conditions to the system domain.

\subsection{Mean flow field}

The displacement thickness $\delta_{\mathrm{s}}^{\prime}$ at the entrance $x_{\mathrm{s}}^{\prime}$ (from the origin of the boundary layer) of the flow domain modelled provides the characteristic length scale and the freestream flow speed, $U_{\infty}^{\prime}$, gives the characteristic speed (hence the characteristic time is $\left.\delta_{\mathrm{s}}^{\prime} / U_{\infty}^{\prime}\right)$. The local $R e_{x}$ at a position $x$ of the flow field relates to the $R e_{\mathrm{s}}$ at the entrance of the domain through $R e_{x}=\gamma \sqrt{x R e_{\mathrm{s}}}$ wherein $R e_{\mathrm{s}}=\rho_{l}^{\prime} U_{\infty}^{\prime} \delta_{\mathrm{s}}^{\prime} / \mu_{l}^{\prime}$ and $\gamma=1.7208$ for the Blasius boundary layer. The horizontal and vertical velocity components of the mean flow are given by

$$
U_{x}=\frac{\mathrm{d} f}{\mathrm{~d} H} \quad \text { and } \quad U_{z}=\frac{\gamma}{2 \sqrt{x R e_{\mathrm{s}}}}\left(H \frac{\mathrm{d} f}{\mathrm{~d} H}-f\right),
$$

${ }_{257}$ where $H=z /\left(\gamma \sqrt{x / R e_{\mathrm{s}}}\right)$ and $f(H)$ satisfies the Blasius equation,

$$
2 \frac{\mathrm{d}^{3} f}{\mathrm{~d} H^{3}}+\gamma^{2} f \frac{\mathrm{d}^{2} f}{\mathrm{~d} H^{2}}=0
$$

258 subject to the boundary conditions $f(0)=\mathrm{d} f / \mathrm{d} H(0)=0$ and $\mathrm{d} f / \mathrm{d} H \rightarrow 1$ as $H \rightarrow \infty$. 
Starting from the two-dimensional (2D) velocity-vorticity disturbance formulation of the Navier-Stokes equations (Davies \& Carpenter 2001) and retaining only the linear velocity and vorticity terms, the evolution of perturbations to the mean flow is governed by

$$
\begin{aligned}
\frac{\partial \omega_{y}}{\partial t}+u_{x} \frac{\partial \Omega_{y}}{\partial x}+u_{z} \frac{\partial \Omega_{y}}{\partial z}+U_{x} \frac{\partial \omega_{y}}{\partial x}+U_{z} \frac{\partial \omega_{y}}{\partial z} & =\frac{1}{R e_{\mathrm{s}}}\left(\frac{\partial^{2} \omega_{y}}{\partial x^{2}}+\frac{\partial^{2} \omega_{y}}{\partial z^{2}}\right) \\
\nabla^{2} \mathbf{u} & =-\nabla \times\left(\omega_{y} \mathbf{e}_{\mathbf{y}}\right)
\end{aligned}
$$

where mean-flow variables appear in capitals, while perturbations to the mean-flow quantities are in lower case; $u_{x}$ and $u_{z}$ are the horizontal and vertical components of the velocity disturbance, while $\Omega_{y}$ and $\omega_{y}$ are respectively the mean-flow and disturbance vorticity in the direction perpendicular to the $x$ - and $z$-axes. Instead of solving the vector Poisson equation (2.3), we make use of the Helmholtz decomposition (Wu \& Thompson 1973; Kempka et al. 1995) and express the disturbance flow field as the sum of its rotational and irrotational parts respectively constructed using distributions of line-vortex and source-sink elements; thus the perturbation velocity is written as

$$
\begin{aligned}
\mathbf{u}(\mathbf{x})= & \int_{R \neq R_{\mathrm{b}}} \nabla G\left(\mathbf{x}, \mathbf{x}^{\prime \prime}\right) \times \omega_{y}\left(\mathbf{x}^{\prime \prime}\right) \mathbf{e}_{\mathbf{y}} \mathrm{d} R\left(\mathbf{x}^{\prime \prime}\right)+\int_{R_{\mathrm{b}}} \nabla G\left(\mathbf{x}, \mathbf{x}^{\prime \prime}\right) \times \omega_{y}\left(\mathbf{x}^{\prime \prime}\right) \mathbf{e}_{\mathbf{y}} \mathrm{d} R\left(\mathbf{x}^{\prime \prime}\right) \\
& -\int_{S} \sigma\left(\mathbf{x}^{\prime \prime}\right) \nabla G\left(\mathbf{x}, \mathbf{x}^{\prime \prime}\right) \mathrm{d} S\left(\mathbf{x}^{\prime \prime}\right),
\end{aligned}
$$

where, $G=(1 / 2 \pi) \log \left(1 /\left|\mathbf{x}-\mathbf{x}^{\prime \prime}\right|\right)$ is the $2 \mathrm{D}$ infinite domain Green's function and $\sigma$ the strength of the source-sink sheet applied to the flow boundary. We remark that the sourcesink elements on the boundary surface are used to enforce the no-flux condition when the flow is perturbed and therefore their strengths, like those of the line-vortex elements, are variables in the calculation of the perturbed flow field. For arbitrarily deforming walls, the use of source-sink boundary elements to enforce no-flux is far more convenient than the commonly used vortex images in vortex methods. In the above integral expressions the double prime indicates a dummy variable, while $R$ and $S$ respectively denote integration in the fluid domain and on the boundary surface. The rotational part is divided into boundary-flow field, $R_{\mathrm{b}}$, and the interior to this boundary, $R \neq R_{\mathrm{b}}$, contributions in order to apply the tangential and normal boundary conditions at the boundary cells and surfaces.

Following Ehrenstein \& Gallaire (2005), we make use of the Robin boundary conditions at the entrance $x_{\mathrm{s}}$ and exit $x_{\mathrm{o}}$ of the fluid domain

$$
\frac{\partial \omega_{y}}{\partial x}=\mathrm{i} \alpha \omega_{y}, \quad \frac{\partial u_{z}}{\partial x}=\mathrm{i} \alpha u_{z} .
$$

The complex wavenumber $\alpha$ in the above is that of the most unstable Tollmien-Schlichting wave found using the Orr-Sommerfeld equation (over a rigid wall) for a given frequency $\omega_{\mathrm{s}}$ at the entrance and similarly at $\omega_{\mathrm{o}}=\left(R e_{\mathrm{o}} / R e_{\mathrm{s}}\right) \omega_{\mathrm{s}}$ at the exit of the fluid domain. The effect of this choice of $\omega_{\mathrm{s}}$ is discussed in $\S 3$.

The boundary conditions $u_{x}(x, 0, t)=u_{z}(x, 0, t)=0$ are applied at the rigid-wall portions. On the compliant-panel section the velocity and stress components are continuous between fluid and solid. Thus, the linearised boundary conditions for the velocity are 


$$
u_{x}(x, 0, t)+\eta_{z}(x, t) \frac{\partial U_{x}}{\partial z}(x, 0)=\frac{\partial \eta_{x}}{\partial t}(x, t), \quad u_{z}(x, 0, t)=\frac{\partial \eta_{z}}{\partial t}(x, t), x_{\mathrm{cs}} \leqslant x \leqslant x_{\mathrm{co}}
$$

where $\eta_{x}(x, t)$ and $\eta_{z}(x, t)$ are the non-dimensional plate axial and vertical displacement of the compliant-panel, respectively. If the model allows only vertical displacements of the compliant panel, the right-hand side of the first equation of (2.6) becomes zero.

The pressure perturbation (non-dimensionalized using the free-stream dynamic pressure) that drives the compliant-panel motion is obtained by integrating the linearized $z$-momentum equation of the Navier-Stokes equations between the fluid-solid interface and infinity and enforcing that the pressure perturbation vanishes at infinity; thus

$$
\begin{aligned}
p(x, 0, t)= & \int_{0}^{L_{\mathrm{H}}}\left(\frac{\partial u_{z}}{\partial t}+\frac{\partial U_{z}}{\partial x} u_{x}+\frac{\partial U_{z}}{\partial z} u_{z}+U_{x} \frac{\partial u_{z}}{\partial x}+U_{z} \frac{\partial u_{z}}{\partial z}\right) \gamma \sqrt{\frac{x}{R e_{\mathrm{s}}}} \mathrm{d} H \\
& +\int_{0}^{L_{\mathrm{H}}} \frac{1}{R e_{\mathrm{s}}} \frac{\partial \omega_{y}}{\partial x} \gamma \sqrt{\frac{x}{R e_{\mathrm{s}}}} \mathrm{d} H,
\end{aligned}
$$

where $L_{\mathrm{H}}$ is the total height of the computational domain, made large enough to ensure that

$$
\omega_{y}\left(x, L_{\mathrm{H}}, t\right)=0, \quad u_{z}\left(x, L_{\mathrm{H}}, t\right)=0 .
$$

Note that the perturbation pressure, defined by equation (2.7), is dependent upon flowperturbation terms that are themselves dependent upon the boundary-conditions of the deformed wall through equations (2.6).

For the compliant-wall dynamics, we use the one-dimensional beam equation with additional terms to account for a dashpot-type structural damping and a uniformly distributed spring foundation, combined with the normal and the tangential force balance on the compliant wall (Shankar \& Kumaran 2002). Keeping only $O(\epsilon)$ terms, we obtain,

$$
\begin{gathered}
\boldsymbol{n} \cdot \boldsymbol{T} \cdot \boldsymbol{n}=-p(x, 0, t)+\frac{2}{R e_{\mathrm{S}}} \frac{\partial u_{z}}{\partial z}(x, 0, t)-\frac{2}{R e_{\mathrm{S}}}\left(\frac{\partial U_{x}}{\partial z}(x, 0)+\frac{\partial U_{z}}{\partial x}(x, 0)\right) \frac{\partial \eta_{z}}{\partial x} \\
=C_{I} \frac{\partial^{2} \eta_{z}}{\partial t^{2}}+C_{D_{z}} \frac{\partial \eta_{z}}{\partial t}+C_{B} \frac{\partial^{4} \eta_{z}}{\partial x^{4}}+C_{K} \eta_{z} \\
\boldsymbol{t} \cdot \boldsymbol{T} \cdot \boldsymbol{n}=\frac{1}{R e_{\mathrm{s}}}\left(\frac{\partial u_{z}}{\partial x}(x, 0, t)+\frac{\partial u_{x}}{\partial z}(x, 0, t)\right) \\
=C_{I} \frac{\partial^{2} \eta_{x}}{\partial t^{2}}+C_{D_{x}} \frac{\partial \eta_{x}}{\partial t}-C_{A} \frac{\partial^{2} \eta_{x}}{\partial x^{2}}
\end{gathered}
$$

where, $\boldsymbol{n}=-\left(\partial \eta_{z} / \partial x\right) \boldsymbol{e}_{\boldsymbol{x}}+\boldsymbol{e}_{\boldsymbol{z}}, \boldsymbol{t}=\boldsymbol{e}_{\boldsymbol{x}}+\left(\partial \eta_{z} / \partial x\right) \boldsymbol{e}_{\boldsymbol{z}}$ are the unit vectors normal and the tangential to the compliant panel respectively, and $\boldsymbol{T}=-P \boldsymbol{I}+\left(1 / R e_{\mathrm{s}}\right)\left[\nabla \boldsymbol{U}+(\nabla \boldsymbol{U})^{T}\right]$ is the total stress tensor of the fluid on the compliant wall. The non-dimensional coefficients of inertia, damping, flexural rigidity, in-plane stiffness (the resistance of the compliant wall to the change of the axial deformations) and spring-foundation stiffness respectively, are defined by 


$$
\begin{array}{r}
C_{I}=\frac{\rho_{\mathrm{m}}^{\prime} h_{\mathrm{m}}^{\prime}}{\rho_{\mathrm{l}}^{\prime} \delta_{\mathrm{s}}^{\prime}}, \quad C_{D_{z}}=\frac{D_{z}^{\prime}}{\rho_{\mathrm{l}}^{\prime} U_{\infty}^{\prime}}, \quad C_{D_{x}}=\frac{D_{x}^{\prime}}{\rho_{\mathrm{l}}^{\prime} U_{\infty}^{\prime}}, \\
C_{B}=\frac{B^{\prime}}{\rho_{\mathrm{l}}^{\prime} U_{\infty}^{\prime}{ }^{2} \delta_{\mathrm{s}}^{\prime 3}}, \quad C_{A}=\frac{E^{\prime} h_{\mathrm{m}}^{\prime}}{\left(1-\nu^{2}\right) \rho_{\mathrm{l}}^{\prime} U_{\infty}^{\prime} \delta_{\mathrm{s}}^{\prime}}, \quad C_{K}=\frac{K^{\prime} \delta_{\mathrm{s}}^{\prime}}{\rho_{\mathrm{l}}^{\prime} U_{\infty}^{\prime 2}},
\end{array}
$$

with $\rho_{\mathrm{m}}^{\prime}$ and $h_{\mathrm{m}}^{\prime}$ being the material density and thickness respectively, and $B^{\prime}=$ $E^{\prime} h_{\mathrm{m}}^{\prime} /\left[12\left(1-\nu^{2}\right)\right]$ wherein $E^{\prime}$ is the elastic modulus and $\nu$ the Poisson ratio of the wall material.

Hinged boundary conditions are applied at the leading and trailing edges of the compliant panel, hence

$$
\begin{array}{r}
\eta_{z}\left(x_{\mathrm{cs}}, t\right)=\eta_{z}\left(x_{\mathrm{co}}, t\right)=\eta_{x}\left(x_{\mathrm{cs}}, t\right)=\eta_{x}\left(x_{\mathrm{co}}, t\right)=0, \\
\frac{\partial^{2} \eta_{z}}{\partial x^{2}}\left(x_{\mathrm{cs}}, t\right)=\frac{\partial^{2} \eta_{z}}{\partial x^{2}}\left(x_{\mathrm{co}}, t\right)=0 .
\end{array}
$$

It is noted that since we investigate the stability of high Reynolds-number flow over a compliant panel the terms in the left-hand side of the equation (2.9) which feature $R e_{\mathrm{s}}$ in the denominator make a small contribution to the normal force balance, however they are included for the sake of the model completeness. Finally, the tangential-force balance, equation (2.10), becomes redundant in the case of the model where only vertical structural displacements are allowed.

\subsection{Eigenproblem formulation}

We proceed by applying the decomposition,

$$
\begin{array}{r}
\left\{\omega_{y}, \sigma, u_{x}, u_{z}\right\}(x, z, t)=\left\{\hat{\omega}, \hat{\sigma}, \hat{u}_{x}, \hat{u}_{z}\right\}(x, z) \exp (\lambda t), \\
p(x, 0, t)=\hat{p}(x, 0) \exp (\lambda t), \quad \eta_{x}(x, t)=\hat{\eta}_{x}(x) \exp (\lambda t), \quad \eta_{z}(x, t)=\hat{\eta}_{z}(x) \exp (\lambda t),
\end{array}
$$

where $\lambda=-i \omega$, together with the complex conjugate part of the eigen-decomposition, ${ }_{328}$ to the linear system of equations (2.2), (2.4), (2.9) and (2.10), taking into account the boundary conditions (2.5)-(2.8) and (2.13), to transform it to the generalized eigenvalue problem

$$
\boldsymbol{C}_{\mathbf{2}} \hat{\boldsymbol{x}}=\lambda \boldsymbol{C}_{\mathbf{1}} \hat{\boldsymbol{x}}, \quad \hat{\boldsymbol{x}}=\left\{\hat{\omega}, \hat{\sigma}, \hat{\eta}_{x}, \hat{\phi}_{x}, \hat{\eta}_{z}, \hat{\phi}_{z}\right\}^{T},
$$

with $\hat{\phi}_{x}=\lambda \hat{\eta}_{x}, \hat{\phi}_{z}=\lambda \hat{\eta}_{z}$ from which the eigenvalues $\lambda$ and eigenvectors $\hat{\boldsymbol{x}}$ can be extracted. If the real part of an eigenvalue $\lambda$ is positive, temporal instability occurs, whereas a negative real part indicates that disturbances decay with time. We remark that the system equation (2.15) is smaller than that which would ensue if the corresponding Poisson equation were solved, since in the present method $\hat{\sigma}$ is evaluated only on the boundary.

\subsection{Local stability analysis}

A Chebyshev collocation matrix combined with the companion matrix method (Bridges \& Morris 1984; Danabasoglu \& Biringen 1990) is implemented to solve the local spatial eigenvalue problem for the complete spectrum. Details of the formulation are given in the Appendix A. 329 330 331 
We restrict the transient analysis to the model which allows only vertical displacements of the compliant wall, even though the analysis could easily be extended to accommodate two degrees of freedom. In order to investigate the transient behavior of the FSI system we adopt standard methods, for example see Schmid (2007) and Coppola \& de Luca (2010), but define the energy norm for the present FSI system to be

$$
\begin{aligned}
E(t)= & \frac{1}{2} \int_{0}^{L_{\mathrm{H}}} \int_{0}^{L}\left(\left|u_{x}\right|^{2}+\left|u_{z}\right|^{2}\right) \mathrm{d} x \mathrm{~d} H \\
& +\frac{1}{2} \int_{0}^{L_{\mathrm{c}}}\left(C_{I} \dot{\eta}_{z}{ }^{2}+C_{B}\left(\frac{\partial^{2} \eta_{z}}{\partial x^{2}}\right)^{2}+C_{K} \eta_{z}^{2}\right) \mathrm{d} x,
\end{aligned}
$$

where the flow kinetic energy is evaluated by the first integral on the right-hand side and the kinetic and strain energies of the compliant panel are captured by the second integral. We look for an initial disturbance which maximizes the energy at time $t$, i.e.

$$
G(t)=\max _{\boldsymbol{x}_{0} \neq 0} \frac{\|\boldsymbol{x}(t)\|_{E}^{2}}{\left\|\boldsymbol{x}_{0}\right\|_{E}^{2}},
$$

in which the disturbances are constructed by the linear superposition of the two- ${ }_{352}$ dimensional temporal modes (Ehrenstein \& Gallaire 2005; Åkervik et al. 2007) and therefore

$$
\boldsymbol{x}(x, H, t)=\sum_{j=1}^{N u m} k_{j}(t) \hat{\boldsymbol{x}}_{j}(x, H),
$$

where Num is the number of converged global eigenvalues used. Taking into account that they must satisfy the initial-value form of the system (2.15), the maximum energy growth becomes

$$
G(t)=\left\|\boldsymbol{F} \exp (\Lambda t) \boldsymbol{F}^{-1}\right\|_{2}^{2},
$$

with $\Lambda=\operatorname{diag}\left[\lambda_{1}, \lambda_{2}, \ldots, \lambda_{N u m}\right]$ and $\boldsymbol{R}=\boldsymbol{F}^{T} \boldsymbol{F}$ the Cholesky decomposition of the 358 Gramian matrix $\boldsymbol{R}$ with entries

$$
\begin{aligned}
R_{i j}= & \frac{1}{2} \int_{0}^{L_{H}} \int_{0}^{L}\left(\hat{u}_{x, i}^{*} \hat{u}_{x, j}+\hat{u}_{z, i}^{*} \hat{u}_{z, j}\right) \mathrm{d} x \mathrm{~d} H \\
& +\frac{1}{2} \int_{0}^{L_{c}} C_{I} \dot{\hat{\eta}}_{z, i}^{*} \dot{\hat{\eta}}_{z, j}+C_{B}\left(\frac{\partial^{2} \hat{\eta}_{z}^{*}}{\partial x^{2}}\right)_{i}\left(\frac{\partial^{2} \hat{\eta}_{z}}{\partial x^{2}}\right)_{j}+C_{K} \hat{\eta}_{z, i}^{*} \hat{\eta}_{z, j} \mathrm{~d} x,
\end{aligned}
$$

where ${ }^{*}$ denotes the complex conjugate. The largest growth at time $t$ is then given by 360 the largest singular value of $\boldsymbol{F} \exp (\Lambda t) \boldsymbol{F}^{-1}$ and the initial condition that provides it is ${ }_{361}$ given by $\boldsymbol{F}^{-1} \boldsymbol{z}$, with $\boldsymbol{z}$ being the right singular vector.

\subsection{Numerical solution}

A second-order finite-difference method is used for discretisation in the $x$-direction both for the linearized Navier-Stokes and compliant-wall equations and a Chebyshev pseudospectral method (Canuto et al. 1988; Baltensperger \& Trummer 2002) is applied in the 
$z$-direction. This has been shown to give sufficient accuracy for this type of FSI stability problem (Davies \& Carpenter 1997b) provided that sufficient resolution of the domain is applied; details showing that we meet this requirement are provided where we present the results of this paper. The flow domain is discretized into $M=M_{\mathrm{w} 1}+M_{\mathrm{c}}+M_{\mathrm{w} 2}$ cells in the streamwise direction, where $M_{\mathrm{w} 1}, M_{\mathrm{c}}$ and $M_{\mathrm{w} 2}$, are respectively the number of fluid cells over the upstream rigid-wall, the compliant-panel and the downstream rigid-wall sections, while $N+1$ points are deployed in the $z$-direction with the following transformation used to map the collocation points from the interval $[1,0]$ onto $\left[0, L_{\mathrm{H}}\right]$.

$$
H_{k}=L_{H}\left[1-\cos \left(\frac{k \pi}{2 N}\right)\right] \quad \text { for } \quad k=0,1 \ldots N .
$$

Then, the discretized form of the momentum equation $(2.2)$ for each cell $(i, j)$ is written 375 for example as

$$
\begin{aligned}
-\lambda \hat{\omega}_{i j}= & \left.\left.\frac{\partial \Omega_{y}}{\partial x}\right|_{i j} \hat{u}_{x}\right|_{i j}+\left.\left.\frac{\partial \Omega_{y}}{\partial z}\right|_{i j} \hat{u}_{z}\right|_{i j}+\left.U_{x}\right|_{i j} \frac{3 \hat{\omega}_{i j}-4 \hat{\omega}_{i(j-1)}+\hat{\omega}_{i(j-2)}}{2 \Delta x} \\
& +\left.U_{z}\right|_{i j} \sum_{k=1}^{N} D_{i j, k j} \hat{\omega}_{k j}-\frac{1}{R e_{\mathrm{S}}}\left(\frac{\hat{\omega}_{i(j+1)}-2 \hat{\omega}_{i j}+\hat{\omega}_{i(j-1)}}{(\Delta x)^{2}}+\sum_{k=1}^{N} D_{i j, k j}^{2} \hat{\omega}_{k j}\right) \\
& \text { for } \quad i=2 \ldots N-1, j=2 \ldots M-1,
\end{aligned}
$$

where $\boldsymbol{D}$ and $\boldsymbol{D}^{2}$ (written above in tensor form) are the Chebyshev differentiation matrices (Baltensperger \& Trummer 2002) which generate the first and second derivative at the collocation points, respectively. In the above expression, a second-order backward finitedifference scheme has been utilized for discretisation of the advection term, while secondorder central finite-differences are used for the diffusion term in the streamwise direction.

The Helmholtz decomposition, equation (2.4), is approximated by zero-order vortex sheets and zero-order source sheets (Houghton \& Carpenter 2003; Katz \& Plotkin 1991), which for each cell are written as

$$
\begin{aligned}
\left.\hat{u}_{x}\right|_{i j}= & \sum_{k=1}^{N} \sum_{l=1}^{M} I_{i j, k l}^{V X} \hat{\omega}_{k l}+\sum_{l=1}^{M} I_{i j, 1 l}^{P X} \hat{\sigma}_{1 l}+\sum_{l=1}^{M} I_{i j, N l}^{P X} \hat{\sigma}_{N l} \\
& +\sum_{k=2}^{N-1} I_{i j, k 1}^{P X} \hat{\sigma}_{k 1}+\sum_{k=2}^{N-1} I_{i j, k M}^{P X} \hat{\sigma}_{k M}, \\
\left.\hat{u}_{z}\right|_{i j}= & \sum_{k=1}^{N} \sum_{l=1}^{M} I_{i j, k l}^{V Z} \hat{\omega}_{k l}+\sum_{l=1}^{M} I_{i j, 1 l}^{P Z} \hat{\sigma}_{1 l}+\sum_{l=1}^{M} I_{i j, N l}^{P Z} \hat{\sigma}_{N l} \\
& +\sum_{k=2}^{N-1} I_{i j, k 1}^{P Z} \hat{\sigma}_{k 1}+\sum_{k=2}^{N-1} I_{i j, k M}^{P Z} \hat{\sigma}_{k M}, \\
& \text { for } i=1 \ldots N, j=1 \ldots M,
\end{aligned}
$$

where $\boldsymbol{I}^{V X}, \boldsymbol{I}^{V Z}, \boldsymbol{I}^{P X}$ and $\boldsymbol{I}^{P Z}$ are the influence coefficient matrices, which give the $x \quad 385$ and $z$ components of the rotational and irrotational velocity components at the $(i, j) \quad 386$ cell due to the presence of a vortex sheet at $(k, l)$ cell and source sheet at the boundary surface respectively. For completeness, expressions for the influence coefficients are given in Appendix B. 
Finally, the ARPACK library (Lehoucq et al. 1998) has been used to extract a significant part of the spectrum of equation (2.15), namely 3000 eigenvalues and their respective eigenvectors, using a relatively large Krylov subspace of 9000 vectors.

\section{Results and discussion}

We focus on the global stability of system modes arising from each of the well-known travelling-wave flutter (TWF) and Tollmien-Schlichting Waves (TSWs) that have been predicted to occur in Blasius boundary-layer flow over compliant walls using a local analysis. All the results presented in the following sections have been obtained using the compliant-wall model that allows only vertical displacements except in sub-section 3.2 , where results from global and local stability analyses using the axial-vertical displacement model are presented. We choose the wall parameters in such a way that the critical velocity for the onset of divergence instability in potential flow over a finite length compliant wall (Garrad \& Carpenter 1982; Pitman \& Lucey 2009) is above the freestream flow speed $U_{\infty}^{\prime}=10 \mathrm{~m} / \mathrm{s}$ used herein as a base case. Throughout the results, the fluid is water with density $1000 \mathrm{~kg} / \mathrm{m}^{3}$ and dynamic viscosity $1.37 \times 10^{-3} \mathrm{Ns} / \mathrm{m}^{2}$. The Reynolds number at the entrance to the computational domain, $R e_{\mathrm{s}}$, is set to 3000 for the eigen-analysis, and to 1000 for the transient analysis.

Three types of compliant panels are used herein, termed wall-1, wall-2 and wall-3, with the base values of their physical properties listed in Table 1. Also included in Table 1 are the values of their corresponding non-dimensional parameterst. Wall-1 is typical of the Kramer-type wall studied in Carpenter \& Garrad (1985) that was capable of transition delay but with a reduced elastic modulus $E^{\prime}$. For this wall, divergence onset first occurs at a flow speed $U_{\infty}^{\prime}=10.32 \mathrm{~m} / \mathrm{s}$ with critical wavelength $\lambda^{\prime}=0.0044 \mathrm{~m}$ as determined using the potential-flow theory of Carpenter \& Garrad (1986). The wall length (0.04 $\mathrm{m})$ is chosen so that it is substantially longer than both this critical wavelength and that of TWF predicted by local-stability theory and shown later. Accordingly, the FSI system features the dynamics of an infinitely-long compliant coating most often studied in stability analyses; however, its fixed ends remain crucial in the global stability analysis presented in $\S 3.1 .1$.

Wall-2 is chosen so that the frequencies of its in vacuo structural modes in vertical displacements,

$$
\omega_{n}=\left(\frac{C_{B}\left(n \pi / L_{c}\right)^{4}+C_{K}}{C_{I}}-\frac{C_{D_{z}}^{2}}{4 C_{I}^{2}}\right)^{\frac{1}{2}},
$$

where $n$ is the mode number, are close to those of the range of unstable TSWs in the boundary layer when the panel has length $0.04 \mathrm{~m}$. Using the finite-wall potential-flow analysis of Garrad \& Carpenter (1982), divergence-onset occurs at the critical flow speed $U_{\infty}^{\prime}=13.67 \mathrm{~m} / \mathrm{s}$ in the fundamental panel mode, $n=1$. Because the contribution of its flexural rigidity to overall wall stiffness far exceeds that of its spring foundation, its dynamics are very similar to those of a simple flexible plate (panel) for which the wavelength of the lowest-frequency mode is determined by the panel length. Wall-3 is of a similar type to wall-1 but it has been made stiffer so that the FSI system is free of the TWF instability and its structural eigenfrequencies are beyond the range of those of unstable TSWs while its divergence-onset flow speed is $U_{\infty}^{\prime}=22.8 \mathrm{~m} / \mathrm{s}$ and the

$\dagger$ The effect of parametric variations from the base values within these wall types are also investigated 
Table 1: Base values of the physical properties and non-dimensional parameters of the systems studied.

\begin{tabular}{lllll}
\hline Panel property & $\begin{array}{l}\text { Symbol } \\
\text { (units) }\end{array}$ & wall-1 & wall-2 & wall-3 \\
& & & & \\
\hline Elastic modulus & $E^{\prime}\left(\mathrm{N} / \mathrm{m}^{2}\right)$ & $1.0 \times 10^{4}$ & $5.0 \times 10^{6}$ & $1.0 \times 10^{5}$ \\
Spring coefficient & $K^{\prime}\left(\mathrm{N} / \mathrm{m}^{3}\right)$ & $115 \times 10^{6}$ & $10 \times 10^{6}$ & $400 \times 10^{6}$ \\
Thickness & $h_{\mathrm{m}}^{\prime}(\mathrm{m})$ & $2 \times 10^{-3}$ & $4 \times 10^{-3}$ & $2 \times 10^{-3}$ \\
Density & $\rho_{\mathrm{m}}^{\prime}\left(\mathrm{kg} / \mathrm{m}^{3}\right)$ & 1000 & 1000 & 1000 \\
Length of compliant section & $L_{\mathrm{c}}^{\prime}(\mathrm{m})$ & $0.04,0.01^{\dagger}$ & $0.01-0.05$ & 0.01 \\
Upstream rigid section & $L_{\mathrm{w} 1}^{\prime}(\mathrm{m})$ & 0.01 & $0.06-L_{\mathrm{c}}^{\prime} / 2$ & 0.01 \\
Downstream rigid section & $L_{\mathrm{w} 2}^{\prime}(\mathrm{m})$ & 0.01 & $0.06-L_{\mathrm{c}}^{\prime} / 2$ & 0.01 \\
& & & & \\
\hline
\end{tabular}

\begin{tabular}{lllll}
\hline $\begin{array}{l}\text { Non-dimensional } \\
\text { parameter }\end{array}$ & Symbol & wall-1 & wall-2 & wall-3 \\
& & & & \\
\hline Reynolds number & $R e_{\mathrm{s}}$ & $3000,1000^{\dagger}$ & 3000 & 1000 \\
Inertia & $C_{I}$ & $4.87,14.6^{\dagger}$ & 9.73 & 14.6 \\
Damping & $C_{D_{z}}$ & $0-0.2,0-1^{\dagger}$ & $0-0.5$ & $0-0.5$ \\
Flexural rigidity & $C_{B}$ & $1.28,34.6^{\dagger}$ & 5127.7 & 345.7 \\
In-plane stiffness & $C_{A}$ & 0.649 & 648.8 & 19.5 \\
Spring stiffness & $C_{K}$ & $0.473-3.355,0.158^{\dagger}$ & 0.041 & 0.548 \\
& & & & \\
\hline
\end{tabular}

${ }^{\dagger}$ values for transient analysis using wall-1 physical data

wavelength of the critical mode is $\lambda^{\prime}=0.008 \mathrm{~m}$. For all walls, the effect of structural damping, $D_{z}^{\prime}$, in the range 0 to $10^{4} \mathrm{Ns} / \mathrm{m}^{3}$, is studied in order to assess this as a means to control system instabilities or reduce transient growth.

Validations of predicted eigenvalues and their corresponding eigen-vectors using the present modeling have been undertaken using appropriate comparisons with localstability analyses in the literature, for example Carpenter \& Morris (1990). Our local-stability results have then been used to construct the spatial amplification of convectively unstable TSWs over a compliant panel in order to create benchmarks against which the spatial amplification computed using the present methods have been compared.

Figure 2(a) shows the full eigenvalue spectrum using the wall-1 data while the detail of figure 2(b) shows the effect of two variations from the base data that increase the structural stiffness or incorporates structural damping. The horizontal axis, $\omega_{r}$, gives the oscillatory part of each mode, while the vertical axis, $\omega_{i}$ gives its temporal growth rate, positive values indicating amplification while negative values indicate decay. Convergence of the global spectrum was achieved (for example, see Tsigklifis \& Lucey (2015)) with 240 uniformly distributed grid points in the streamwise direction and 75 collocation points in the vertical direction for height of the computational domain $L_{\mathrm{H}}=30$. In the streamwise discretisation, 160 points were used for the compliant-wall section giving a 


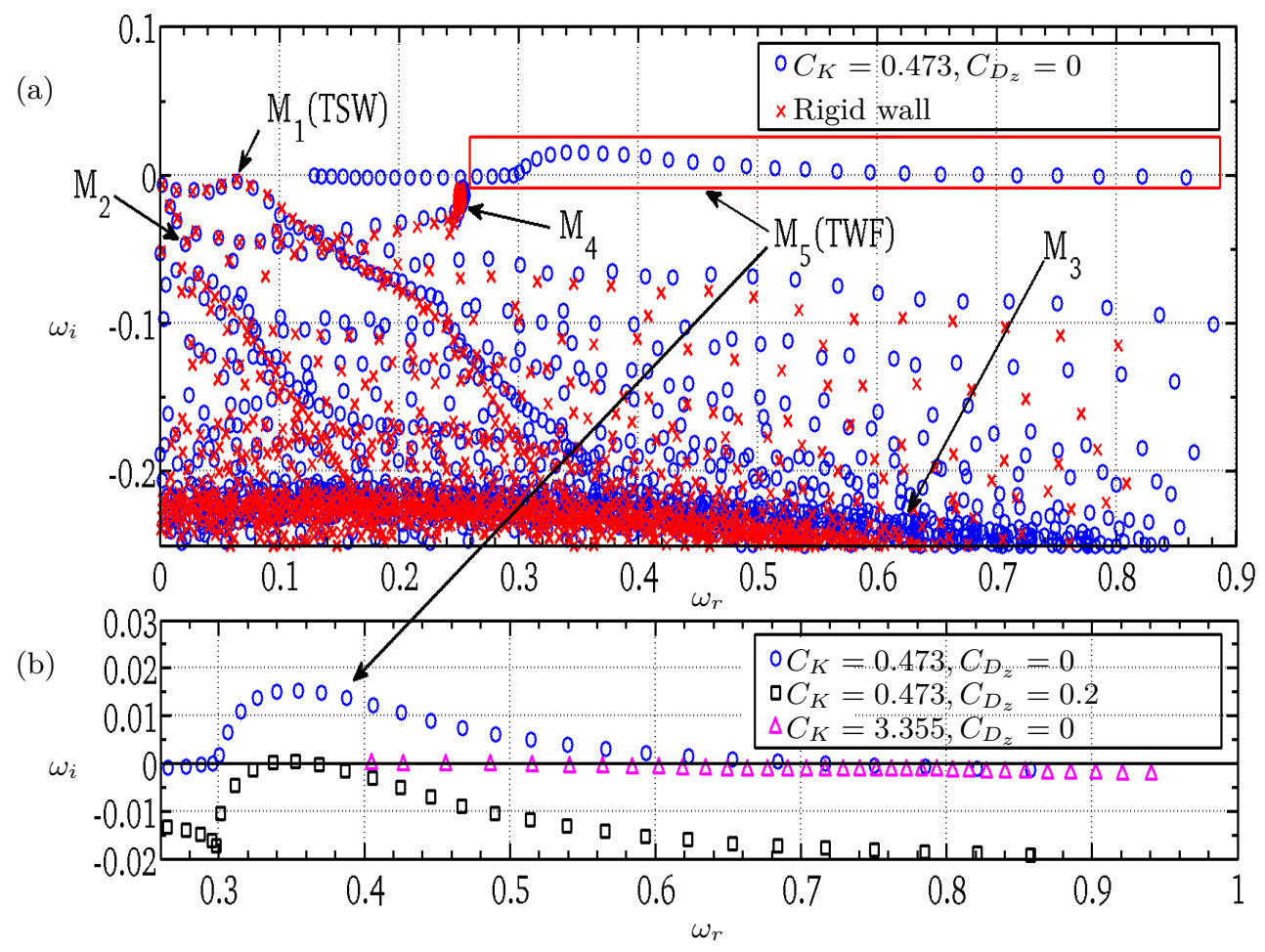

Figure 2: (a) Eigenvalue spectrum at $R e_{\mathrm{s}}=3000$ from global-stability analysis for wall1 data and rigid wall at $x_{\mathrm{s}}=1012$ and frequency of the inlet boundary condition $\omega_{\mathrm{s}}=0.07755$, (b) Detail from (a) to show the $\mathrm{M}_{5}$ branch and its variation for different structural damping and stiffness. (The meanings of the mode-branch labels $\mathrm{M}_{1}-\mathrm{M}_{5}$ are provided in the text.)

dimensionless element length $\Delta x=0.6$, which is sufficient to resolve the wavelength of the most unstable TWF mode (17 grid points) and of the associated structural mode (13 grid points) predicted by local-stability analysis. Five mode types are identified in this figure: $M_{1}$ is the TSW branch that for these system properties is seen to be stable, $M_{2}$ is the Orr-mode branch, $\mathrm{M}_{3}$ is the continuous spectrum of the Orr-Sommerfeld equation while $\mathrm{M}_{4}$ are modes associated with the Orr-Sommerfeld entry boundary conditions. It is noted that all of these branches can be identified with the corresponding spectrum for a rigid wall that is also plotted in figure 2(a). The fifth, $\mathrm{M}_{5}$, is the TWF branch that is seen to be unstable over a range of oscillation frequencies. Clearly, there is no rigid-wall analogue for this flexible-wall-based mode in figure 2(a). However, it is seen in figure 2(b) that either a suitable amount of stiffening or the inclusion of sufficient structural damping stabilises the TWF branch. Finally, we have varied both the length of the rigidwall regions upstream and downstream of the compliant panel and the choice of inlet and outlet disturbance frequencies to the flow domain, used to generate complex $\alpha$ in equation (2.5) to ensure that this type of modal-interaction behaviour is not an artifact of the finite computational domain. 
Herein we present predictions of the time-asymptotic behaviour of the system (i.e. solutions of the boundary-value problem) that characterise the system state after transient responses to the initiation of system disturbances have either been wholly attenuated or convected away from the region of the compliant panel. The main focus is upon the findings of the global-stability analysis arising from the decomposition of equations (2.14) that leads to the eigen-problem of equation (2.15). However, we also perform local analyses wherein all perturbations are proportional to $\exp [\mathrm{i}(\alpha x-\omega t)]$ in which $\alpha=\alpha_{r}+\mathrm{i} \alpha_{i}$ is the complex wavenumber that arises from solving the Orr-Sommerfeld equation (A 2), for a given frequency $\omega$. Clearly this type of analysis uses the assumption of a compliant panel that is infinitely long within a boundary layer of fixed displacement thickness determined by the value of the local Reynolds number at the mid-chord of the panel, and its formulation only permits spatial growth or decay $\left(\alpha_{i}<0\right.$ and $\alpha_{i}>0$ respectively, for downstream-propagating modes) of system disturbances.

\subsubsection{Travelling-wave flutter (TWF) branch}

Using local analyses, TWF on a compliant wall of infinite extent has been shown to be a convective instability (Carpenter \& Garrad 1986; Carpenter \& Morris 1990; Dixon et al. 1994; Lucey \& Carpenter 1995). For the definition of convective and absolute instabilities, see Huerre \& Monkewitz (1985, 1990), while Lucey (1998); Lucey \& Peake (2003) describe the application of these concepts when ideal flow interacts with a flexible panel. Unstable TWF waves grow spatially in the downstream direction from a source of applied excitation. The instability arises from the action of the fluid flow on what are essentially structural waves, their growth caused by irreversible transfer of energy from the flow to the flexible wall that occurs when a critical layer exists within the boundary layer. In the wave-classification system of Benjamin (1963), they are denoted Class B.

For a finite panel with wall-1 data, figure 2 (see branch $\mathrm{M}_{5}$ ) shows that TWF can become a global, temporally amplifying, instability that would lead to the destabilisation of the compliant panel at all spatial locations and without a continuing applied source of excitation. We emphasise that the global temporal amplification of this unstable branch is insensitive to changes of the inlet outlet boundary conditions nor increases to the length of the compliant panel. To understand the global destabilisation mechanism, we present in figure 3 the time-evolution of the panel deflection for the most unstable TWFbranch eigenvalue in figure 2. First, it is clearly seen that the amplitude of the mode grows with time $t$ (non-dimensionalised using displacement thickness and free-stream flow speed). Second, is also seen to be spatially amplified by comparing deflection amplitudes near the compliant-panel leading edge $(x=1040$ where the coordinate of location is non-dimensionalised using the displacement thickness) with those near its trailing edge $(x=1130)$. The dashed- and dotted-line envelopes in these figures signify the spatial change in amplitude of the structural (S) and TWF mode respectively predicted by localstability analysis conducted at the complex frequency of the global mode, the results of which are presented in figure 5(a) that will be discussed later. It can be seen that the spatial amplification of the wall displacement in the downstream direction predicted by the global model agrees well with that of the structural mode $(\mathrm{S})$ predicted by a local stability analysis; however, the structural mode is spatially stabilised in the direction of its phase speed and group speed. The spatial amplification of the velocity disturbance away from the wall predicted by the global stability agrees with that of the TWF mode predicted by a local stability analysis (not shown). Finally, it can be seen that the global mode is a combination of two types of wave, the expected downstream-travelling TWF (predicted by a local stability analysis) and an upstream travelling wave. It is this 
(a)

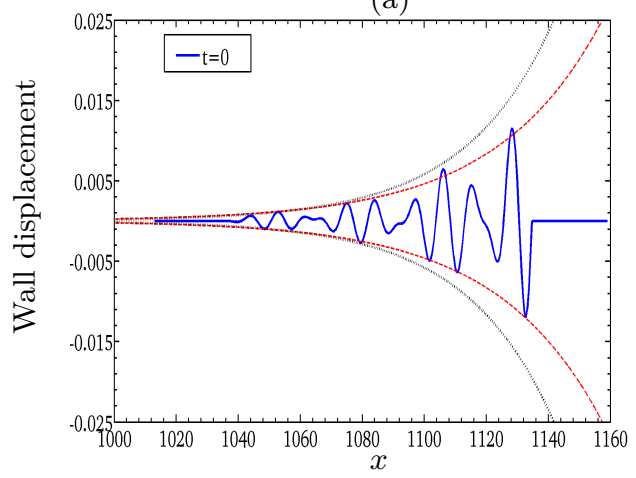

(c)

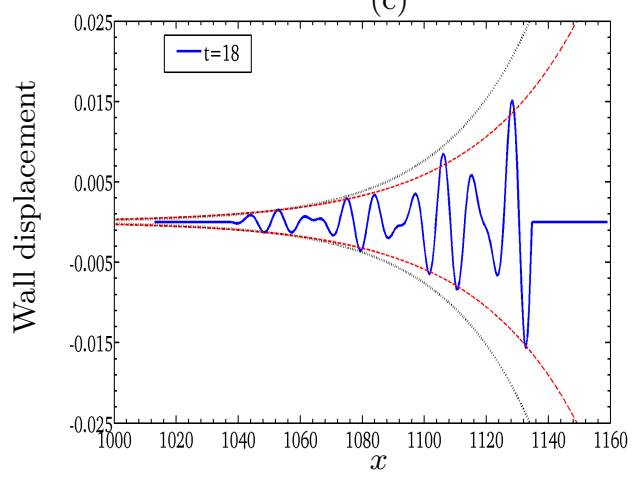

(b)

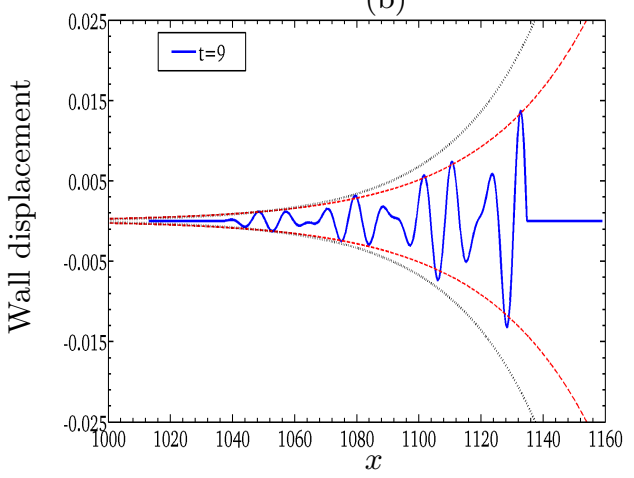

(d)

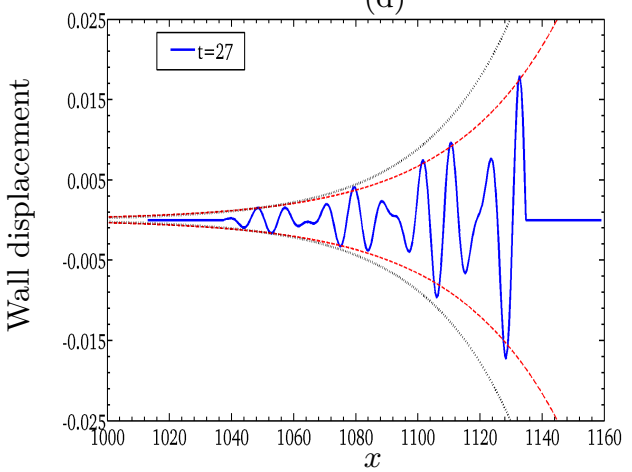

Figure 3: Spatio-temporal evolution of the panel deflection for the most unstable global mode on the TWF-branch $\left(\mathrm{M}_{5}\right)$ in figure 2. The dashed and dotted lines are plots of the spatial amplification of the structural (S) and TWF modes found using a local-stability analysis at the global-mode frequency and shown in figure 5(a) positioned as a best-fit to the global mode. The computational domain extends from $x=1012$ to 1158 (nondimensional distances from the boundary-layer origin) while the compliant panel occupies the region $x=1037$ to 1134 .

combination of waves on a flexible wall with fixed ends, permitting energy propagation in both downstream and upstream directions, that leads to the temporal growth found for the global mode. The TWF transfers flow kinetic energy to the compliant wall as it propagates downstream. At the trailing edge the TWF continuously excites the upstreampropagating structural mode and its arrival at the leading edge of the compliant panel provides a continuous source of excitation for the TWF.

As has been seen in figure 2 the inclusion of sufficient structural damping $C_{D_{z}}$ stabilises the TWF-branch. This might be expected since TWF occurs essentially through the destabilization of what is a wall flexural wave (that exists in vacuo) and it has been categorised as a Class B wave. Stiffening the compliant panel by increasing the spring stiffness $C_{K}$ also exercises a stabilising effect as evidenced in the same figure. Again, this could have been anticipated on the basis of local-stability analyses, given that in the limit of infinite stiffness the wall is rigid and therefore unable to support the flexural waves that are the source of TWF; in fact, when the compliant coating is sufficiently stiff that the speed of its structural waves exceeds that of the free-stream flow (external to the boundary layer) the critical layer ceases to exist.

We now consider the effect of the Reynolds number on the global TWF-mode insta- 


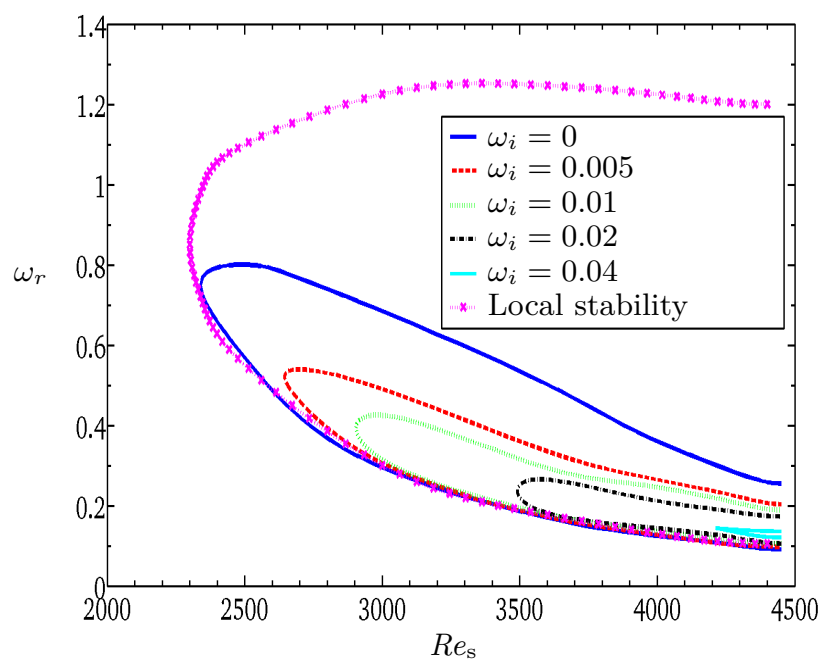

Figure 4: Temporal amplification curves in the $\omega_{r}-R e_{\mathrm{s}}$ plane for the TWF mode predicted by the global stability analysis for wall- 1 data at $x_{\mathrm{s}}^{\prime}=0.416 \mathrm{~m}$ with $R e_{\mathrm{s}}$ varied through the free-stream flow speed. The neutral curve predicted by local spatial stability analysis is also included.

bility. Figure 4 shows its temporal amplifications curves in the $\omega_{r}-R e_{\mathrm{s}}$ plane as predicted by the global stability analysis. For comparison, we have also included the neutral curve predicted by our local spatial-stability analysis. There is a good match between local spatial and global temporal stability regarding the prediction of the critical Reynolds number - the value at which instability first appears with increasing $R e_{\mathrm{s}}$ - and the lower part of the neutral curve but poor agreement for the upper branch. As can be discerned in figure 2 when damping is absent, the TWF branch $\left(\mathrm{M}_{5}\right)$ crosses the real axis as a near asymptote that gives large values of the oscillatory frequency $\omega_{r}$ which correspond to small spatial wavelength. The global stability model is stretched to resolve accurately the small spatial wavelength spectrum due to the use of a second-order finite-difference scheme for streamwise spatial discretisation. However, the discretisation parameters we have used accurately capture the eigenvalues of the main instability loop and the maximum temporal amplification rate because these are located at lower oscillation frequencies.

We now show how the foregoing globally unstable TWF mode branch might manifest itself in a local-stability analysis. Figures 5(a) and (b) are dispersion diagrams, without and with (at $C_{D_{z}}=0.2$ ) structural damping respectively, for wavenumber as a function of real wave frequency; i.e. the results of a spatial-stability analysis. The local analysis is conducted for the Reynolds number obtained using the displacement thickness at the midpoint of the compliant panel. In these figures the real part of the eigenvalue, $\alpha_{r}$, indicating the inverse of wavelength, is plotted as different types of blue lines for the different mode branches with axis scale on the left of the figure. The imaginary part of each eigenvalue, $\alpha_{i}$, that gives the spatial amplification is plotted in red with its axis scale in red on the right of the figure. For a downstream-propagating wave, spatial amplification and decay in the downstream direction are respectively indicated by negative and positive values of $\alpha_{i}$; for upstream-propagating waves, positive and negative values indicate amplification and decay respectively in the upstream direction. The direction of wave travel can be inferred 
from the wave phase speed, $c=\omega_{r} / \alpha_{r}$ and propagation from the sign of group velocity $\left(c_{g}=\partial \omega_{r} / \partial \alpha_{r}\right)$. We have also included the in vacuo dispersion curve for the compliant wall because it is readily seen that the TWF and S mode branches respectively approach those of the downstream and upstream free waves of the wall as the effect of fluid loading steadily reduces with increasing frequency. The different types of blue (real part) and red (imaginary part) markers added into the figures are the complex wavenumbers that result when the complex frequency $\omega=\omega_{r}+\mathrm{i} \omega_{i}$ of the most unstable global mode on the TWF-branch $\left(\mathrm{M}_{5}\right)$ and the least stable global mode on the TSW-branch $\left(\mathrm{M}_{1}\right)$ in figure 2 are used in the local analysis.

In figure 5(a) the local-stability analysis reveals the expected downstream propagating (with positive $\alpha_{r}$ ) TWF branch that amplifies in the downstream direction. Also evident is the upstream-propagating (with negative $\alpha_{r}$ ) structural-mode branch labelled $\mathrm{S}$ that evidences wave travel and attenuation in the upstream direction. These features could be demonstrated formally through the Briggs-Bers technique (for its application to potential flow over a flexible wall see Peake (2004)) or in the analytical categorisation of wave types in Ashpis \& Reshotko (1990) as (ci) in their figure 5 with the compliant-panel trailing edge acting as its source of excitation in the global-stability analysis. Thus, the global mode that contained two wave types in figure 3 may be considered to be the combined effect of these two modes predicted by the local analysis. However, a local analysis alone would not be sufficient to show that these combine to yield a global instability on a panel of finite extent. Moreover, while the wavelengths of these two modes at the complex frequency of the global mode are predicted well by the local-stability analysis, their amplification/decay rates are not; in fact it can be seen that the local stability analysis over-predicts the downstream amplification of the TWF mode while it under-predicts the upstream attenuation of the structural mode. Accordingly, we may conclude that while the local-stability analysis can provide the basis for understanding the interaction of finite compliant panels with a boundary-layer flow, only a global analysis can yield quantitative predictions of system stability. Figure 5(b) shows that structural-damping levels, sufficient to stabilise the global TWF-mode branch (see figure 2(b)), do not eliminate the spatial amplification of the TWF-mode branch that continues to exist as a convectively unstable mode, albeit with reduced spatial-growth rate (comparison of red continuous lines between figures $5(\mathrm{a})$ and (b)). However, the local-stability analysis at the frequency of the most unstable global TWF modes in figure 2 with and without damping predicts only a slight stabilization of the spatial amplification (comparison of the red triangles between figures 5(a) and (b)). Structural damping is also seen to increase the attenuation of the upstream-travelling structural wave $(\mathrm{S})$ in its direction of propagation and this reinforces the interpretation of the global TWF-mode instability of a finite panel as comprising a combination of the TWF and S modes predicted by local-stability analyses.

Turning attention to the globally stable TSW-branch in figure 2, it is seen in figure 5(a) that there exists a range of frequencies for which TSWs are convectively unstable in that they amplify as they propagate downstream. The local analysis conducted at the complex frequency of the least-stable global mode shows (see markers) that a conventional localstability analysis under-predicts the growth rate of the unstable mode. It is also seen by comparing the growth rates of the TSW markers that wall-compliance has a mildly stabilising effect on the convectively unstable TSWs as compared with their amplification over a rigid wall (comparison of the red crosses and squares in figures 5(a)). Comparing figures 5(a) and (b), the spatial growth rate of the TSW is also seen to be slightly increased by the inclusion of damping but the compliant panel continues to exercise a stabilizing effect on the TSW as compared to its growth over a rigid wall. 
Comparing the spatial amplification rates of the most unstable TWF and TSW modes, either for real $\omega$ or the global complex $\omega$, in figures $5(\mathrm{a})$ and (b), it can be seen that the amplification of the convectively unstable TWF mode is greater than the spatial amplification rate of the TSW mode and that structural damping is only marginally attenuating at the global frequency. A compliant panel with these properties could not be used for transition delay because the spatial amplification of the TWF mode exceeds that of TSW and thus it may be TWF that causes transition as has been shown to occur by Lucey \& Carpenter (1995) when modelling the experiments of Gaster (1988). Accordingly, in practical applications of compliant panels for transition delay it is essential to choose compliant-wall properties (a combination of structural stiffness and damping for a given mass ratio $C_{I}$ ) that prevent global stability and postpone the onset and/or reduce the growth rates of convectively unstable TSWs and TWF. The latter has been accomplished in the optimisation of transition delay based upon local stability analyses (Carpenter 1991; Dixon et al. 1994). Accordingly, in what follows we focus on a parametric study of the effects of structural stiffness and damping on the stability of the global TWF-mode.

We first focus upon the relationship between compliant-panel stiffness and the Reynolds number at which global instability on the TWF branch first occurs. To characterise the stiffness, that comprises contributions from both plate flexure and spring foundation, we define a non-dimensional wall stiffness as the ratio of its free-wave speed to the free-stream flow speed, hence

$$
K_{\mathrm{IW}}=\frac{c_{\min .}^{\prime}}{U_{\infty}^{\prime}}=\frac{\left(4 C_{K} C_{B}\right)^{1 / 4}}{C_{I}^{1 / 2}}
$$

We remark that the simple inviscid theory of Carpenter \& Garrad (1986), using a local analysis, predicts $K_{\mathrm{IW}}=1$ as the criterion for TWF-onset on the basis that this value is the threshold between the existence and absence of a critical layer within the boundary layer.

It is well known that the self-similarity of Blasius boundary-layer flow means that its dynamics can be characterised uniquely by the Reynolds number. This continues to be the case for local stability analyses where non-parallel effects are neglected and the compliant-wall is homogenous in the streamwise direction. However, when a panel of finite length is inserted in an otherwise rigid wall, the FSI system becomes inhomogeneous in the streamwise direction. Accordingly for a fluid with given kinematic viscosity, Reynoldsnumber variation can be achieved in one of two distinct ways, either (a) changing the downstream distance of the plate $x_{\mathrm{s}}^{\prime}$ (from the boundary-layer origin) for a fixed free-stream flow speed $U_{\infty}^{\prime}$ or (b) changing the free-stream flow speed $U_{\infty}^{\prime}$ for a fixed position $x_{\mathrm{s}}^{\prime}$ of the compliant panel. These changes do not give equivalent FSI dynamics even though they may result in the same Reynolds number. The former appears more appropriate for theoretical analysis because the dimensionless stiffness depends only on the structural properties, while the latter is more convenient for comparison with experimental measurements obtained at a specific location along the plate.

In figure $6(\mathrm{a})$ we show how the stiffness $K_{\text {IW }}$ at which global TWF-mode instability first appears varies with the Reynolds number for different values of a fixed free-stream flow speed $U_{\infty}^{\prime}$ where the streamwise position, $x_{\mathrm{s}}^{\prime}$ (hence boundary-layer thickness), is varied. Figure $6(\mathrm{~b})$ is the corresponding result but here the flow speed is allowed to vary while different fixed downstream positions $x_{\mathrm{s}}^{\prime}$ are considered. These results have been generated using the local spatial stability analysis and have been verified a posteriori with the global stability, since, as can be seen in figure 4, there is good agreement 
(a)

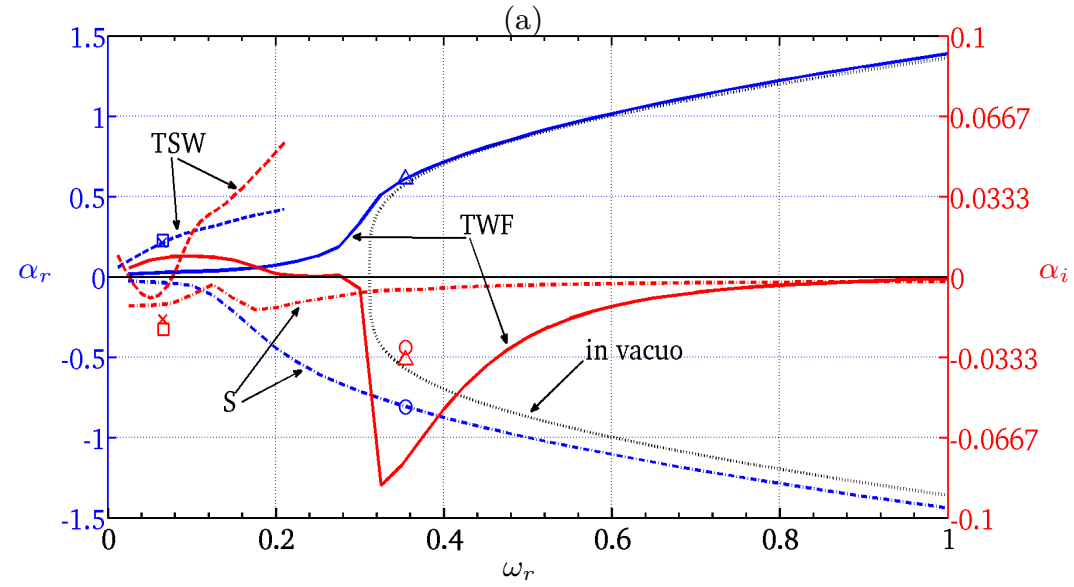

(b)

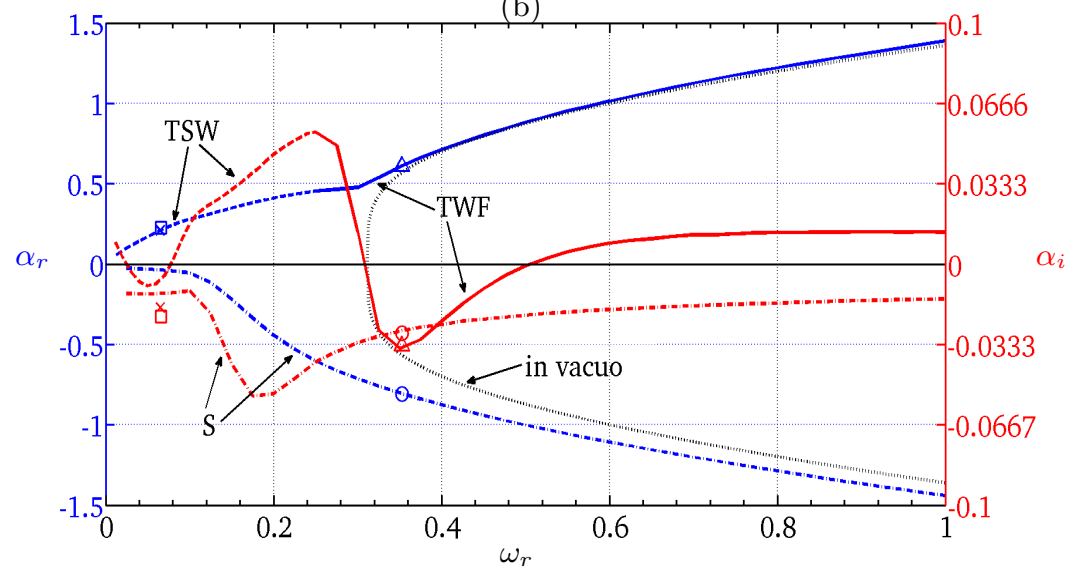

Figure 5: Dispersion diagrams showing the variation of complex wavenumber, with real (blue) and imaginary (red) parts respectively on left and right vertical axes, with wave frequency for (a) $C_{D_{z}}=0$ and (b) $C_{D_{z}}=0.2$. Discrete-data markers indicate the complex eigenvalues from the local-stability analysis conducted at the complex frequencies of the most unstable TWF global mode (triangles and circles respectively for TWF and $\mathrm{S}$ modes) and the least stable global TSW mode (crosses and squares respectively for compliant and rigid walls) of figure 2 . The grey line is the dispersion curve for free waves of the compliant-wall.

between local and global stability analyses for the lower branch of the neutral curve and the lowest Reynolds number at which the global TWF-branch features instability. These results indicate that the FSI system becomes more stable with respect to global TWF modes with increasing downstream distance from the leading edge of the plate at a given free-stream flow speed $U_{\infty}^{\prime}$, or as the free-stream flow speed increases for a given position of the plate. Moreover, figure $6(\mathrm{a})$ shows that, at any given location of the flexible panel, a higher flow speed is destabilising and this can be expected since the ratio of wall-stiffness to flow stiffness (that scales with the dynamic pressure of the free-stream flow) is reduced; i.e. the wall appears softer. Figure 6(b) shows that, for any given flow speed, the flexible panel is more stable when located further downstream where the boundary layer is thicker via higher $x_{\mathrm{s}}^{\prime}$ and/or lower Reynolds number (free-stream 
flow speed for the variation used in $6(\mathrm{~b}))$. It is noted that $K_{\mathrm{IW}}$ can exceed unity but this does not mean that the critical layer does not exist. The approximate threshold $K_{\mathrm{IW}}=1$ discussed below equation (3.2) above is based upon the assumption of a thin boundary layer and its formulation does not account for the effects of fluid inertia on the compliant-wall wave speed.

Figure 7(a) confirms the finding that the FSI system becomes more stable with increased downstream location of the flexible panel for a given free-stream flow speed $U_{\infty}^{\prime}$. In this figure we plot the temporal eigenvalue spectrum from the global-stability analysis for different Reynolds numbers while maintaining a constant free-stream flow speed $U_{\infty}^{\prime}=10 \mathrm{~m} / \mathrm{s}$. The temporal growth rates of the TWF modes reduce with increasing downstream location because the wavelength of the instability becomes smaller relative to the length of the compliant wall and therefore the finiteness effect of the compliant panel that underpins the global instability is reduced.

The principal mechanism of the TWF instability is the phase shift in the pressure perturbation across the critical layer which gives rise to irreversible energy transfer to the wall (Carpenter \& Morris 1990; Carpenter \& Gajjar 1990). In order to reveal why increasing the downstream distance of the flexible panel from the origin of the boundary layer is stabilising for the global TWF mode, we use a local stability analysis to calculate the rate of work done by the pressure disturbance on the wall (Carpenter \& Morris 1990),

$$
p \frac{\partial \eta_{z}}{\partial t} \sim \int_{0}^{L_{H}} \operatorname{Re}\left[\left(\mathrm{i}\left(\alpha U_{x}-\omega\right) \hat{u}_{z}-\frac{1}{R e_{x}}\left(\frac{\mathrm{d}^{2}}{\mathrm{~d} z^{2}}-\alpha^{2}\right) \hat{u}_{z}\right) \hat{u}_{z}(H=0)\right] \mathrm{d} H=\int_{0}^{L_{H}} S \mathrm{~d} H,
$$

having integrated the $z$-component of linearized Navier-Stokes equations from the compliant wall through to the free stream to obtain the pressure perturbation on the wall. Figure $7(\mathrm{~b})$ shows the distribution of the function $S$ through the boundary layer for the cases shown in figure $7(\mathrm{a})$. It can be seen that as the boundary layer progresses downstream both the positive rate of work above the critical layer and the dissipation in the viscous wall layer become smaller in magnitude but the total contribution is in favour of the global TWF-mode stabilisation. As the boundary layer progresses downstream, its thickness increases and the critical layer, where the fluid flow speed equals the free wave speed, moves away from the wall and its effect is attenuated.

We now consider the effect of structural damping in conjunction with compliant-wall stiffness variations. We define the non-dimensional damping, based upon the dynamics of the compliant-wall in vacuo, as

$$
D_{\mathrm{IW}}=\frac{D_{z}^{\prime} c_{\min }^{\prime}}{\rho_{1}^{\prime} U_{\infty}^{\prime 2}}=\frac{C_{D_{z}}\left(-C_{D_{z}}^{2}+4 C_{I} C_{K}\right)^{1 / 4} C_{B}^{1 / 4}}{C_{I}^{3 / 4}}
$$

noting that the physical damping is constrained by $C_{D_{z}}<2 \sqrt{C_{I} C_{K}}$; i.e. lower than critical damping for the wall structure.

Figures 8(a) and (b) plot the variation with Reynolds number of the minimum value of damping $D_{\mathrm{IW}}$ required to stabilise all modes on the global TWF branch in figure 2 for different values of fixed free-stream flow speeds and fixed positions of the flexible panel plate respectively, and different values of the foundation-spring stiffness. These results have been generated through the combined use of global and local stability analyses using the following procedure. For given Reynolds number and compliant-wall stiffness we conduct a global stability analysis without structural damping. This result is then used to conduct a local spatial-stability analysis at the predicted complex frequency $\omega=\omega_{r}+\mathrm{i} \omega_{i}$ with the maximum temporal amplification $\omega_{i}$, increasing the structural 
(a)

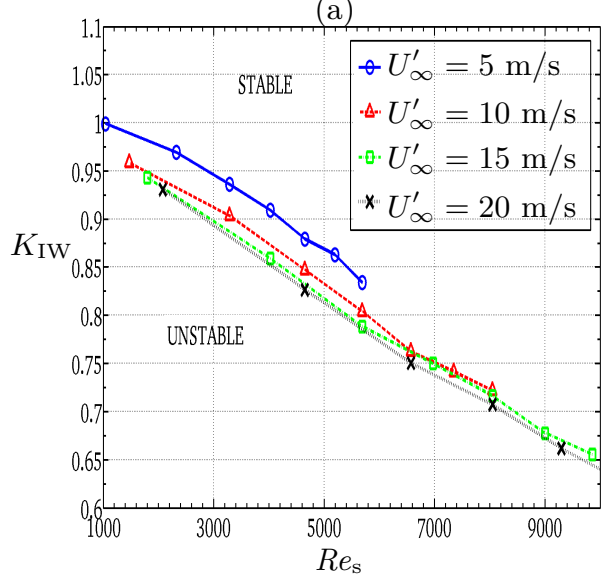

(b)

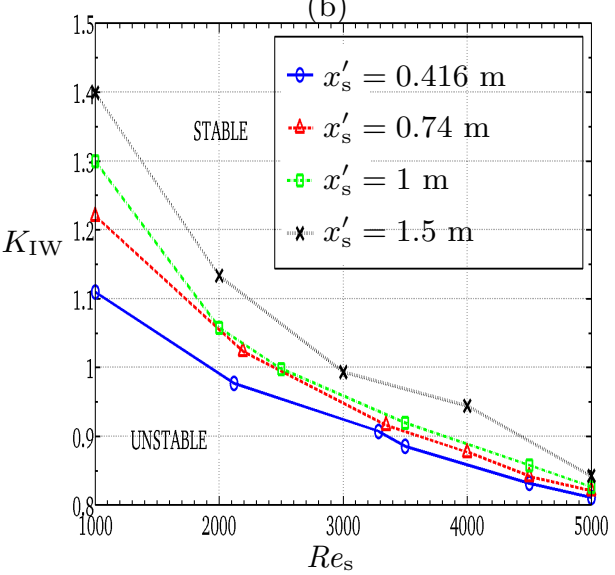

Figure 6: Variation of structural stiffness at which global TWF-mode instability first occurs with Reynolds number through (a) Variation of the distance of the flexible panel from the boundary-layer origin for different fixed free-stream flow speed flow speeds, $U_{\infty}^{\prime}$, and (b) Variation of the free-stream flow speed for different fixed distances $x_{\mathrm{s}}^{\prime}$.
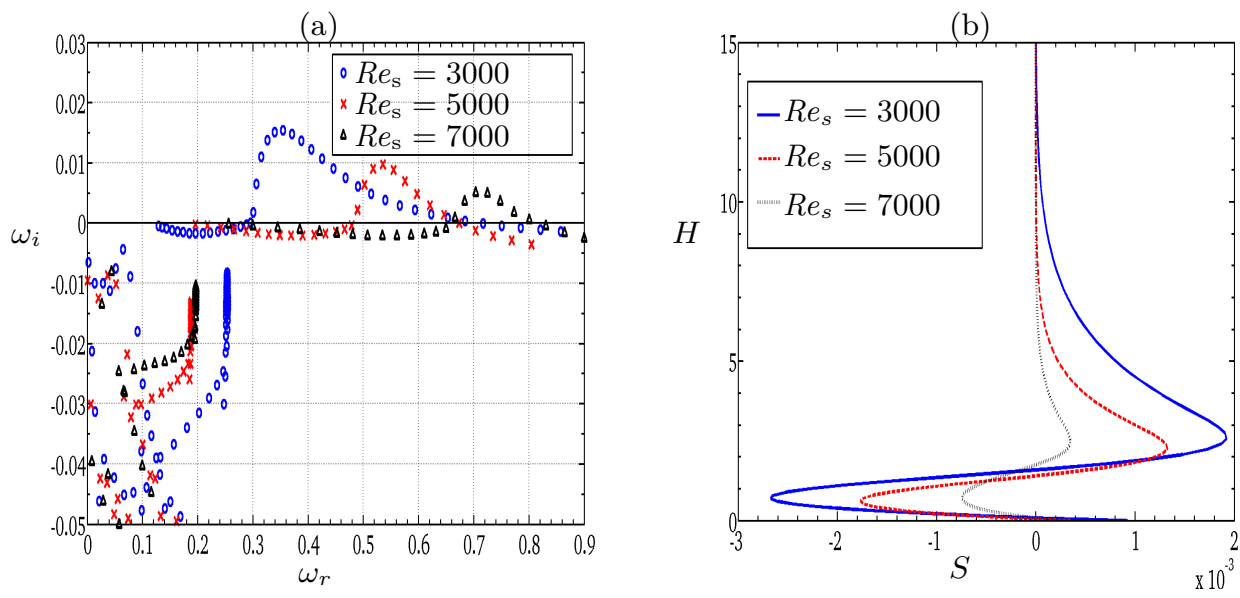

Figure 7: (a) Eigenvalue spectrum from global-stability analysis for different Reynolds numbers and fixed free stream speed $U_{\infty}^{\prime}=10 \mathrm{~m} / \mathrm{s}$. (b) Distribution of the energyproduction function $S$ through the boundary layer for the cases shown in (a) produced by local-stability analysis using wall-1 properties.

damping until the spatial amplification $\alpha_{i}$ crosses the real axis and becomes positive, indicating a spatially stable mode. For this value of the structural damping, we then perform the global-stability analysis to verify that this value of damping marginally stabilises the TWF global-instability branch. This approach has been adopted to reduce the number of computationally expensive global calculations. From the results in figures 8 (a) and (b), it can be seen that there is a good agreement between local and global stability analyses except for cases where edge effects of the finite compliant wall are non-negligible and a higher value of structural damping is needed. Overall, figures 8(a) and (b) show that the level of damping required to eliminate the global TWF-mode instability reduces as the boundary layer progresses downstream or with an increase to 

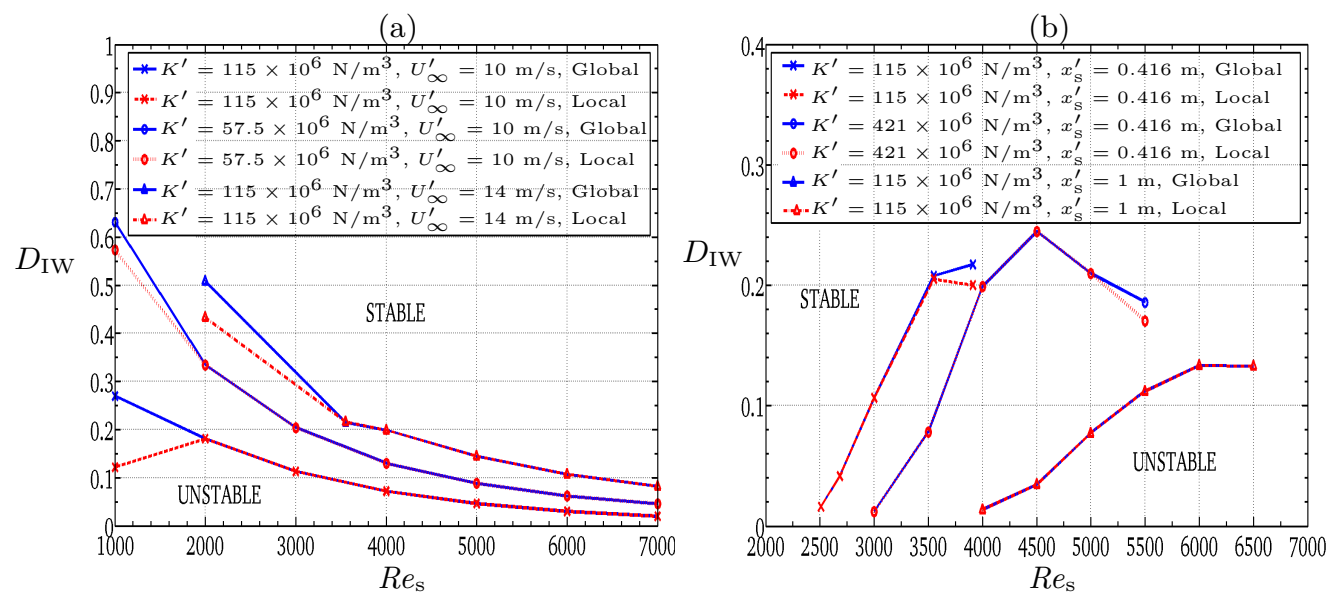

Figure 8: Variation of structural damping at which global TWF-mode instability first occurs with Reynolds number using both global and local stability analyses, for different values of foundation-spring stiffness $\left(K^{\prime}\right)$, through (a) Variation of the distance of the flexible panel from the boundary-layer origin for different fixed free-stream flow speeds, $U_{\infty}^{\prime}$, and (b) Variation of the free-stream flow speed for different fixed distances $x_{\mathrm{s}}^{\prime}$.

the compliant-wall stiffness and/or the decrease of the free-stream flow speed. These findings align with those of figures $6(\mathrm{a})$ and (b), because the global TWF instability can be stabilized by each of increased structural stiffness and damping or a combination thereof.

\subsubsection{Tollmien-Schlichting Wave (TSW) branch}

Local stability analyses of Blasius boundary-layer flow over a compliant wall (Carpenter \& Garrad 1985, 1986; Carpenter 1990), show that TSWs are convective instabilities and Class A waves in the energy classification of Benjamin (1963). In the results of $§ 3.1 .1$ the choice of wall-1 properties rendered the system globally stable for the TSW-branch modes.

Throughout this section we use the properties of wall-2 (listed in table 1) to show that TSWs can combine with structural modes of the finite panel to generate global instability. Figure 9(a) shows one part of the full eigenvalue spectrum for different levels of discretisation for height of the computational domain, $L_{\mathrm{H}}=50$. In the upper panel we increase the number of Chebyshev collocation points in the wall-normal direction for a fixed number of points (120) in the streamwise direction and then, in the lower panel, we increase the number of points in the streamwise direction for a fixed number of collocation points in the wall-normal direction (85). It is seen that for a fairly narrow range of mode frequencies, $\omega_{r}$, the temporal growth rate, $\omega_{i}$, is positive and that the mesh $168 \times 85$ accurately captures the system behavior of the most unstable modes. The 112 grid points $(\Delta x=0.87)$ used for the discretization of the compliant-wall section are able to capture the characteristics of the most unstable TSW (33 grid points per wavelength) and of the associated structural mode (90 grid points per wavelength) in the global destablisation. Compared to the growth rates of unstable modes on the TWF-branch, for example see figure 2 , these rates are very low, being one order of magnitude smaller. However, as a temporal instability, it will come to dominate the system behaviour with the passage of sufficient time. We also note that both its amplification rate and oscillation frequency are sensitive to the change of the frequency of disturbances introduced as entry conditions 
upstream of the compliant panel. This means that any mode on the TSW branch could potentially resonate with a structural mode provided the frequency of the latter falls within the range of the TSW branch. The upper panel of figure 9(b) shows the global eigenmode of the most unstable mode in figure $9(\mathrm{a})$ through its $\hat{u}_{x}$ distribution while the lower panel shows its variation through one vertical slice at the position marked by the vertical dashed line in the upper panel. Clearly, this mode has the well-known characteristics of a Tollmien-Schlichting wave, albeit modified by its interaction with the compliant panel, the extent of which is denoted by the vertical solid lines. Also plotted in the lower panel is the eigenfunction of the TSW predicted by the local spatial-stability analysis at the Reynolds number of the vertical dashed line. While the global and local modes have the same essential characteristics, it is evident that quantitative differences exist between the predictions of the types of analysis.

The mechanism for global instability arises through the interaction of the fluid-based TSW mode and a mode of the wall structure. This can be inferred from figure 9(c) that shows the eigenvalue spectrum for different (non-dimensional) wall lengths. The very short panels, $L_{\mathrm{c}}=24.3$ and 36.5 yield a globally stable system whereas for $L_{\mathrm{c}}=$ 48.7 instability appears at a single system frequency. The shorter panels have structural frequencies, given by (3.1) with its dependence upon $L_{\mathrm{c}}$, that, even for the first panel mode $(n=1)$ are higher than those of the TSW branch. Increasing the length of the panel for fixed structural properties lowers its natural frequencies into the range for which the unstable resonances seen in figure 9(c) can occur. A further increase to the panel length $L_{\mathrm{c}}=73.0$ actually reduces the growth rate because, as will be seen later, the wavelength of the structural mode is such that the resonance with the TSW is less exact than that at $L_{\mathrm{c}}=48.7$. Continuing to increase the panel length enables both the first and second structural modes to interact with the TSW to give two unstable global modes at each of $L_{\mathrm{c}}=97.3$ and 121.7. Further increases to panel length would see the third structural mode creating resonance but, it will be seen later, the increased effective flexibility of the panel (as evidenced by its natural frequency) in general renders it susceptible to a stronger divergence instability.

We now demonstrate that structural damping in the panel can be used to suppress global instability of the TSW-branch modes. Figure 9(d) shows the eigenvalue spectrum of the TSW-branch in figure 9 (c) when $L_{\mathrm{c}}=97.3$ for different levels of (non-dimensional) damping coefficient $C_{D_{z}}$. As the level of damping is increased (from zero), the eigenvalues of the unstable modes move downwards into the negative $\omega_{i}$ plane thereby stabilizing the mode. Although local analyses (Carpenter \& Garrad 1985; Dixon et al. 1994; Lucey \& Carpenter 1995; Carpenter et al. 2001) show that structural damping is spatially destabilising for TSWs in an infinite domain, in keeping with its Class A classification, it is its effect upon the structural mode that combines with the TSW to create the global temporal instability that results in the overall stabilization of the global mode.

We now examine the extent to which a local spatial-stability analysis can predict the mechanism for global instability of the TSW branch. Figures 10(a) and (b) are dispersion diagrams, without and with (at $C_{D_{z}}=0.5$ ) structural damping respectively, for wavenumber as a function of real wave frequency plotted using the same convention as that used in figure 5. The Reynolds number used is based upon the displacement thickness at the mid-point of the panel used in the generation of the global mode in figure 9 (d). Note that in these figures we have, for clarity, omitted the TWF branch that links to the downstream-propagating in-vacuo mode at high frequencies. The TSW branch appears as spatially amplifying in the downstream direction over the range of frequencies $\omega_{\mathrm{r}}: 0.025 \rightarrow 0.09$. Also evident is the structural-mode branch, labelled S, which is upstream propagating, has upstream directed phase speed and is spatially attenuating in 

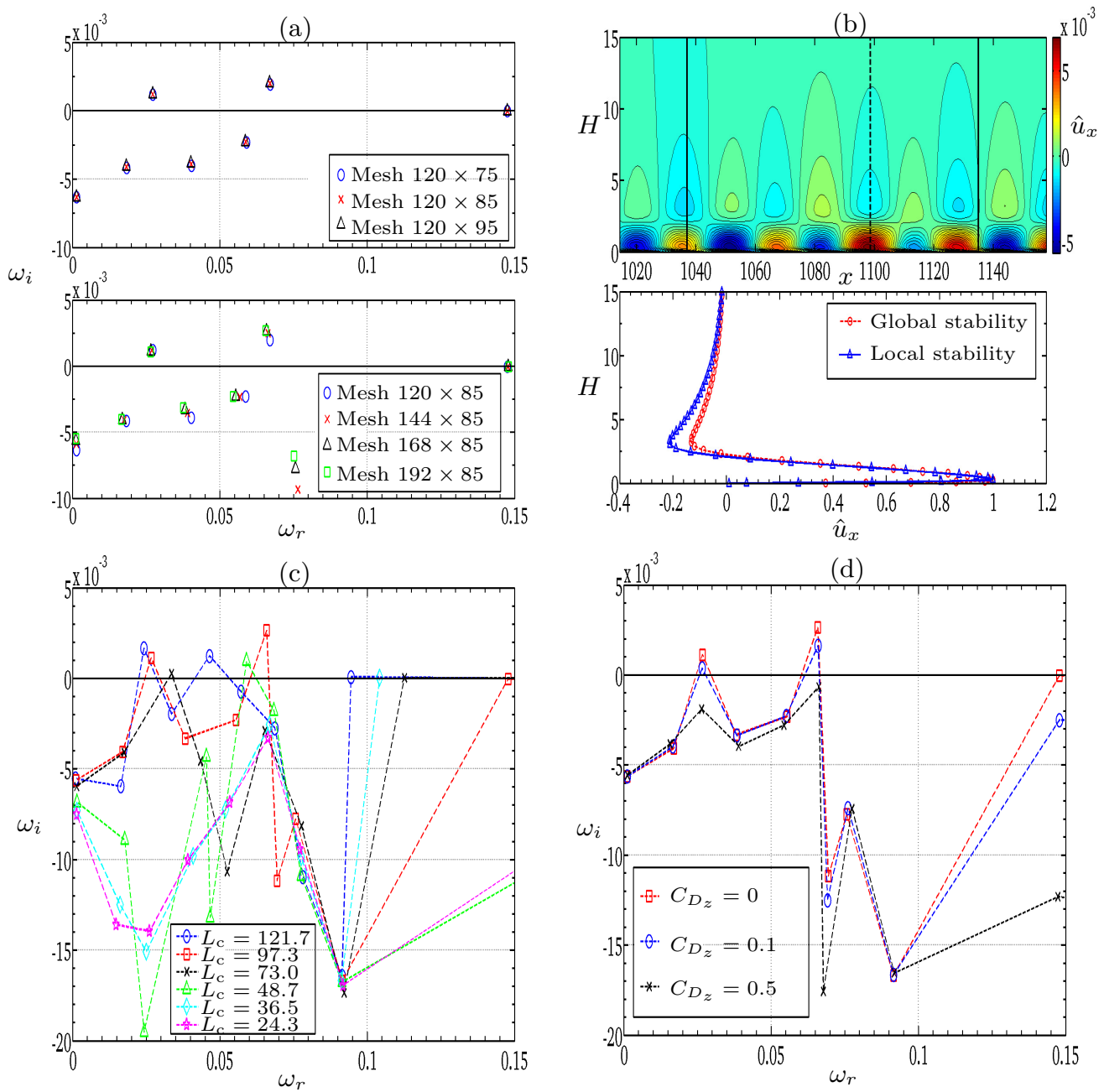

Figure 9: Global-stability analysis using wall-2 data for $x_{\mathrm{s}}=1012$ and at $R e_{\mathrm{s}}=3000$ with frequency of the inlet boundary condition $\omega_{\mathrm{s}}=0.07755$ focusing on the TSW branch. (a) The effect of discretisation for compliant-panel non-dimensional (based upon displacement thickness) length $L_{\mathrm{c}}=97.3$; (b) $\hat{u}_{x}$ distribution (upper panel) of the most unstable global mode in (a) and (lower panel) its comparison with the mode predicted by the local spatial-stability analysis at the position denoted by the dashed line; the solid lines signify the leading and trailing edges of the compliant wall. (c) The effect of panel length on global instability of the TSW branch; the broken lines connecting the discrete eigenvalues (symbols) are sketched in to highlight how growth/decay varies with increasing oscillation frequency for each panel-length case. (d) The effect of structural damping on the global stability of the TSW branch mode for $L_{\mathrm{c}}=97.3$.

the upstream direction. It is these two branches that combine to give the give the global instability presented in figure 9 . However, the local-stability analysis yields a continuous variation of eigenvalues for these two branches because the compliant-wall is assumed to be infinitely long. Accordingly, it is unable to identify the discrete frequencies, determined by compliant-panel length, at which resonance between the two modes might occur. The 
(a)

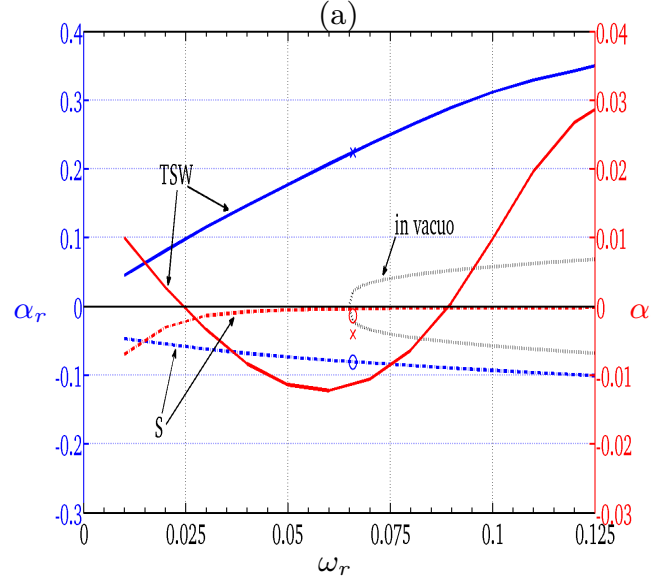

(b)

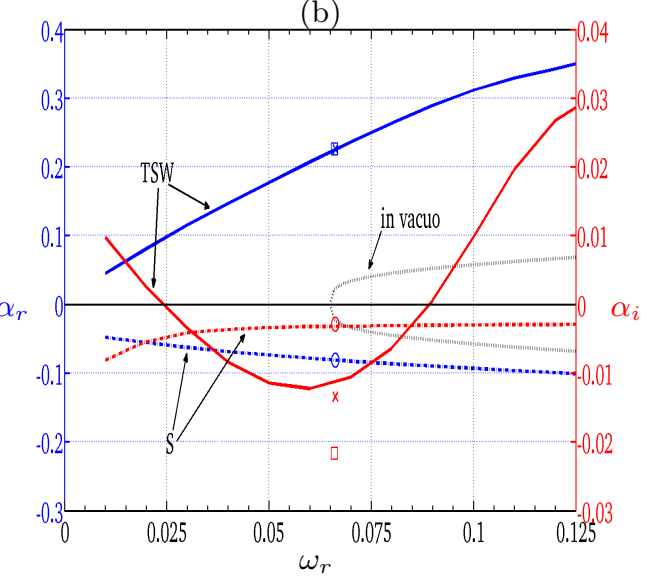

Figure 10: Dispersion diagrams showing the variation of complex wavenumber, with real (blue) and imaginary (red) parts respectively on left and right vertical axes, with wave frequency for (a) $C_{D_{z}}=0$ and (b) $C_{D_{z}}=0.5$. Discrete-data markers indicate the complex eigenvalues from the local-stability analysis conducted at the complex frequency of the most unstable TSW global mode (crosses and circles respectively for TSW and S modes) of figure 9 (d) while the squares give the corresponding eigenvalue for a rigid wall. The grey line is the dispersion curve for free waves of the compliant-wall. Note that the TWF branch has been omitted for clarity.

markers in figures 10(a) and (b) are the local-stability eigenvalues calculated at the complex frequency of the most unstable global mode in figure $9(\mathrm{~d})$, i.e. for panel length $L_{\mathrm{c}}=97.3$. It is seen that these align fairly closely with the predictions for real $\omega$ in the dispersion diagrams. While the level of structural damping $C_{D_{z}}=0.5$ was sufficient to stabilise the TSW global mode as shown in figure $9(\mathrm{~d})$, it is marginally destabilising for the convectively unstable TSW branch in figure 10(b). This is to be expected given that TSW is a Class A instability. However, the local-stability analysis does indicate that damping has a strong stabilising effect on the structural mode, $\mathrm{S}$, and this reinforces the explanation as to why damping can stabilise the globally unstable TSW mode. Finally, even with damping present, figure 10(b) shows that the spatial amplification rate of the convectively unstable TSW over the compliant wall is lower than that which occurs over a rigid wall. Accordingly compliant panels could be designed with sufficient structural damping to suppress the global instability and reduce the growth of TSWs as they travel downstream.

In figure 11(a) we show the dependence of the resonant frequency and the maximum growth rate of the globally unstable TSW mode on the Reynolds number for the properties of wall-2 and fixed compliant-panel length $L_{\mathrm{c}}^{\prime}=0.04 \mathrm{~m}$ at given position $x_{\mathrm{s}}^{\prime}=0.416 \mathrm{~m}$. These dimensional values are provided because changing the Reynolds number (through flow speed for a fixed location) results in changes to the non-dimensional (based upon displacement thickness) flexible-panel length and its distance from the origin of the boundary layer. The wall mode shapes at three Reynolds numbers of the variation are depicted in figure 11(b) noting that increasing the Reynolds number moves the nondimensional location of the flexible panel to greater distances from the boundary-layer origin as reflected in this figure. Broadly, the effect of increasing the Reynolds number is analogous to increasing the length of the compliant panel (shown in figure 9(c) and 
discussed above). This is because increasing the flow speed reduces the panel stiffness relative to the flow stiffness that is proportional to its dynamic pressure. Accordingly, figure 11(a) shows that increasing the Reynolds number reduces the frequency of the fundamental structural mode - labelled MODE 1, the wall shape of which is characterised by the result at $R e_{\mathrm{s}}=2683$ in figure 11(b) that yields the resonance with a TSW. With further increases to $R e_{\mathrm{s}}$, it is the second structural mode, labelled MODE 2, that combines with the TSW to create the most unstable global mode. This pattern of increasingly higher structural modes yielding the most unstable global mode continues with the appearance of the branch and wall mode labelled MODE 3 in figures 11(a) and (b). However, it is seen that for $R e \geqslant 3795\left(U_{\infty}^{\prime}=16 \mathrm{~m} / \mathrm{s}\right)$ a low-frequency divergence-instability mode (labelled D) appears that is insensitive to the inlet-outlet boundary conditions of the flow. The divergence mode has increasing amplification rate and decreasing frequency as $R e_{\mathrm{S}}$ increases and would dominate (over the global TSWmode instability) the system response at sufficiently high Reynolds numbers.

The appearance of divergence in the present system is unsurprising because increasing $R e_{\mathrm{s}}$ through the flow speed, increases the flow stiffness and when this exceeds the panel stiffness, divergence, which is a buckling-type of instability, may occur. It is upon this basis that potential-flow modelling (Garrad \& Carpenter 1982) can be used to predict its onset flow speed. Thus, the black dashed vertical line in figure 11(a) indicates the critical Reynolds number, $R e_{\text {crit. }}=3508\left(U_{\infty}^{\prime}=13.67 \mathrm{~m} / \mathrm{s}\right)$ for the onset of divergence while its mode shape is shown in figure 11(b). Its wavelength (non-dimensionalised by displacement thickness $\delta_{\mathrm{s}}^{\prime}$ ) is approximately $\lambda=148$ at $R e_{s}=3795$ while potential-flow theory predicts $\lambda=227.6$ for the fundamental mode and $\lambda=118.9$ for the second mode at onset flow speeds $U_{\infty}^{\prime}=13.67$ and $14.94 \mathrm{~m} / \mathrm{s}$, respectively. In addition, the wavelength of the least stable global TSW mode for the rigid wall case at $R e_{s}=3795$, is $\lambda=35.2$ and so the critical wavenumber of the divergence instability is smaller than the wavenumber of the least stable TSW mode. This is in agreement with the predictions of Davies \& Carpenter $(1997 a)$ who found that the critical wavenumber of the divergence should be much smaller than the wavenumber of the least stable TSW mode for the divergence instability to be realised.

To conclude this sub-section, we evaluate the levels of structural damping required to stabilise the global TSW mode for the wall-2 properties. Given that low-order structural modes of a finite compliant panel underpin the resonance, as evidenced by Figure 11(b), we define the non-dimensional structural damping in terms of the standing-wave vibrational characteristics of the panel, hence

$$
D_{\mathrm{FW}}=\frac{D_{z}^{\prime} L_{\mathrm{c}}^{\prime} f_{1}^{\prime}}{\rho_{1}^{\prime} U_{\infty}^{\prime 2}}=C_{D_{z}} L_{\mathrm{c}}\left(\frac{C_{B}\left(\pi / L_{c}\right)^{4}+C_{K}}{C_{I}}-\frac{C_{D_{z}}^{2}}{4 C_{I}^{2}}\right)^{\frac{1}{2}},
$$

where $f_{1}^{\prime}$ the eigenfrequency of the fundamental in-vacuo structural mode. In table 2 we list the minimum level of dimensionless structural damping $D_{\mathrm{FW}}$ required to eliminate the unstable resonant behaviour for the three Reynolds-number cases shown in figure 11(b). However, we remark that these findings pertain to the TSWs excited by the specific perturbation $\omega_{\mathrm{s}}=0.05$; to determine the minimum levels of damping for all such global TSW-branch instabilities would require such evaluations across the full range of $\omega_{\mathrm{s}}$ that excite TSW in the FSI system. The fifth and sixth columns in table 2, give the resulting spatial amplification rates of the least stable TSW at the given level of structural damping $D_{\mathrm{FW}}$ for the compliant panel and for a rigid wall. It can be seen that with the required amount of damping to eliminate global instability at each Reynolds number, it is also 

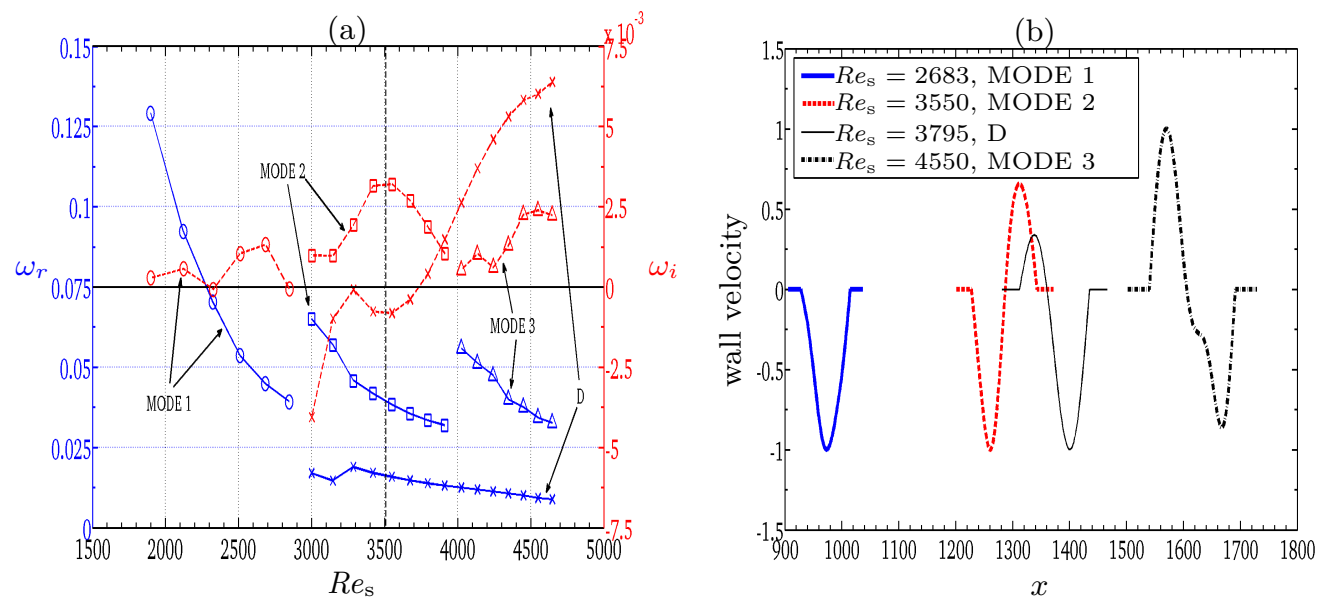

Figure 11: The effect of Reynolds number on (a) the resonant complex frequency of the global instability of the TSW-branch and for wall-2 data for fixed dimensional location based on $x_{\mathrm{s}}^{\prime}=0.416 \mathrm{~m}$, compliant-panel length $L_{\mathrm{c}}^{\prime}=0.04 \mathrm{~m}$ and frequency of the inlet boundary condition $\omega_{\mathrm{s}}=0.05$. (b) shows the normalised mode shapes (through wall vertical velocity) at different Reynolds-number resonances in (a) with the TSW mode and at the onset of the globally unstable divergence (D).

Table 2: Critical damping for stabilisation of the global TSW-branch for the cases shown in figure 11(a) until the onset of the divergence at $R e_{s}=3795$ and corresponding spatial amplification rates $\left(\alpha_{i}\right)$ of the least stable TSW for the compliant and the rigid wall cases.

\begin{tabular}{llllll}
\hline$R e_{\mathrm{s}}$ & $U_{\infty}^{\prime}[\mathrm{m} / \mathrm{s}]$ & $D_{z}^{\prime}\left[\mathrm{kg} /\left(\mathrm{m}^{2} \mathrm{~s}\right)\right]$ & $D_{\mathrm{FW}}$ & Wall-2, $\alpha_{\mathrm{i}}$ & Rigid, $\alpha_{\mathrm{i}}$ \\
\hline 2683 & 8 & 1500 & 1.5696 & $-9.656 \times 10^{-3}$ & $-3.292 \times 10^{-2}$ \\
3550 & 14 & 8200 & 2.2374 & $-1.068 \times 10^{-2}$ & $-2.471 \times 10^{-2}$ \\
3795 & 16 & 10000 & 1.7648 & $-1.087 \times 10^{-2}$ & $-2.477 \times 10^{-2}$ \\
\hline
\end{tabular}

possible to reduce the spatial amplification rates for the least stable TSWs relative to those of the flow over a rigid wall. We also remark that the divergence mode (D), being a Class $\mathrm{C}$ instability, is relatively insensitive to the effect of structural damping. Thus, even though the globally unstable TSW branch can be stabilised through damping, the onset of divergence instability for $R e_{\mathrm{s}} \geqslant 3795$ would render the system globally unstable.

\subsection{Effect of axial and vertical compliant-wall displacements}

Here we investigate the combined effect of axial and vertical structural displacements on the global instabilities of the FSI system. Figure 12 shows a typical spectrum of eigenmodes for wall-1 data from the global-stability analysis which also includes corresponding results for the one degree-of-freedom (vertical) compliant-wall model. The additional axial structural modes are seen to be globally stable. Furthermore, their inclusion does not 


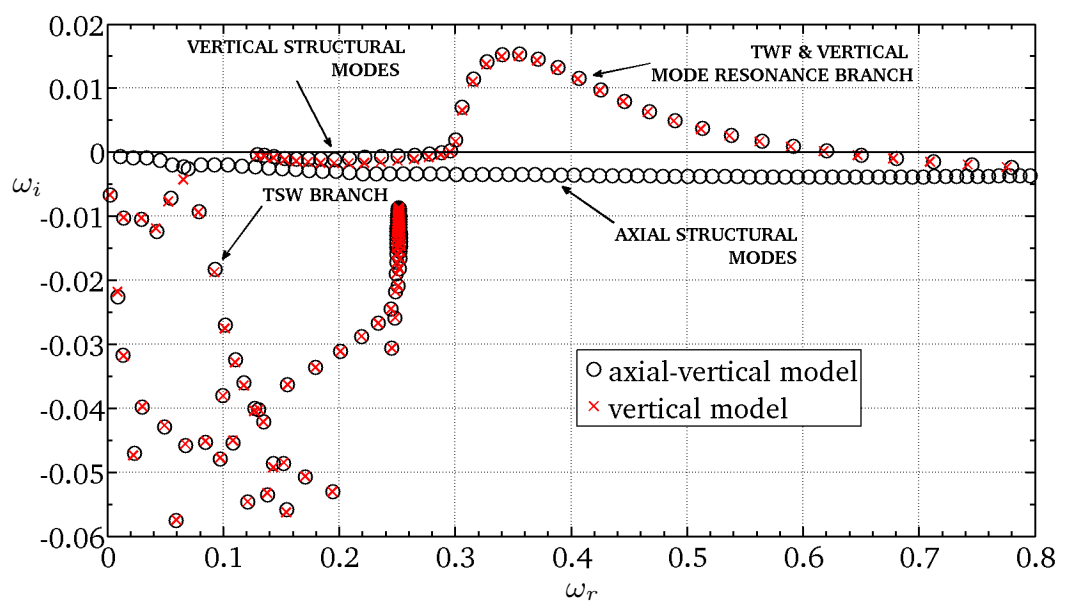

Figure 12: Eigenvalue spectrum at $R e_{\mathrm{s}}=3000$ from global temporal stability analysis of the FSI system for wall-1 data for $x_{\mathrm{s}}=1012$ and frequency of the inlet boundary condition $\omega_{\mathrm{s}}=0.07755$ : Comparison with the model accounting only for the vertical motion.

change the strong global TWF-mode instability predicted by the one-degree-of-freedom (vertical) compliant-wall model investigated in $\$ 3.1 .1$, nor does it generate a globally unstable interaction with the TSW-mode branch. Further results for the wall-2 data (not presented here) indicate that the inclusion of axial wall motion does not alter the globally unstable TSW-resonance investigated in $\$ 3.1 .2$. However, the growth rates of the two globally unstable modes are very marginally increased as can be discerned, for example, through figure 12 by careful inspection of the unstable TWF branch and the least stable mode on the TSW branch. Spatial local-stability analysis of the system that generated figure 12 yields results (not presented here) that are very similar to figure 5 but with the addition of downstream- and upstream-travelling (and propagating) axialmode branches that are both stable in their direction of propagation. The existence of the latter, that transmits energy upstream, makes a small contribution, further to that of the upstream-propagating structural mode (labelled S in figure 5), that combines with TWF and therefore strengthens the global instability mechanism. However, overall, the inclusion of axial-deformation of the compliant wall does not generate significant differences to the FSI behaviour of the system for the parameter-ranges considered in the present paper.

\subsection{Transient growth}

We now assess whether transient growth would be a significant effect in the destabilisation of the finite compliant panels considered in this paper. We consider two types of panel, namely the potentially transition-delaying compliant coating with a reduced stiffness represented by the wall-1 data in table 1 that was found to be susceptible to a global instability of the TWF branch in $\S 3.1 .1$, and a stiffer coating represented by wall-3 data in table 1 that is free from global instability. The relatively low Reynolds number, $R e_{\mathrm{s}}=1000$ with a free-stream flow speed $U_{\infty}^{\prime}=10 \mathrm{~m} / \mathrm{s}$, is used herein.

Figure 13 shows the time history maximum energy growth $G(t)$ for the compliant-wall properties of wall-1 and wall-3 for different levels of structural damping and for a rigid wall. First it is seen that a compliant panel free from global instability (wall-3) produces 


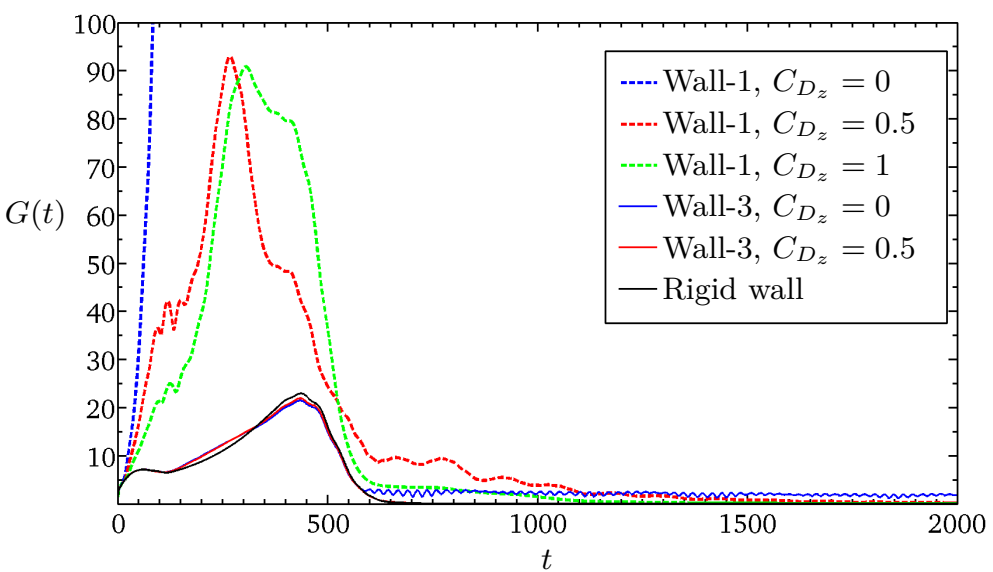

Figure 13: Maximum growth of fluid-structure system energy $G(t)$, as a function of time for two compliant panels with and without structural damping, $C_{D_{z}}$ for $R e_{\mathrm{s}}=1000$, free-stream flow speed $U_{\infty}^{\prime}=10 \mathrm{~m} / \mathrm{s}$ and frequency of the inlet boundary condition $\omega_{\mathrm{s}}=0.07755$.

marginally lower maximum total energy (TE) than the rigid wall. However, it is seen that the compliant panel with wall-1 properties supports very significant levels of transient growth. In the absence of structural damping, the panel experiences global instability and thus its energy time series grows to become infinite. When structural damping at $C_{D_{z}}=0.5$ is used to suppress the global instability, as described in section $\$ 3.1 .1$, the maximum energy is finite but at a much higher level than that for a rigid wall. The inclusion of a higher level of damping, $C_{D_{z}}=1.0$, marginally reduces the peak energy level but increases the temporal width of the energy footprint. Accordingly, transient growth needs to be considered as a factor in the design of compliant panels for transition postponement even if their properties have been tailored to preclude the existence of global instabilities.

To understand better the above behavior but also to calculate the maximum flow kinetic energy which is convected downstream and the effect of structural wall damping on this, we decompose the time evolution of the system total energy (TE) that arises from the optimal initial disturbances (plotted in figure 13) and defined by equation (2.16), into its contributions from the flow kinetic (FKE), structural kinetic (SKE) and structural strain energy (SPE). This is shown in figures 14(a) and (b) for wall-1 and wall-3 properties respectively; for the purpose of comparison, the total energy (TE) trace for the rigid-wall case is included in each of these figures. The overall feature of these results is that the accumulation of flow-kinetic energy dominates transient growth of disturbances.

Figure 14(a) shows that while the fluid kinetic energy is the dominant energy term, the wall-energy terms are non-negligible. The effect of structural damping is seen to reduce the latter terms. This is consistent with earlier results showing that damping can be used to control both global and local TWF modes. However, increasing the damping leads to an increase in the fluid kinetic energy because it has been seen that it has a destabilising effect on the flow-based modes that principally comprise TSWs. Thus, while the inclusion of structural damping is essential to preclude global instability it does lead to larger downstream transfer of fluid kinetic energy through transient growth. In contrast, the stiffer wall-3 that was chosen to be free of globally unstable modes without the need 
(a)

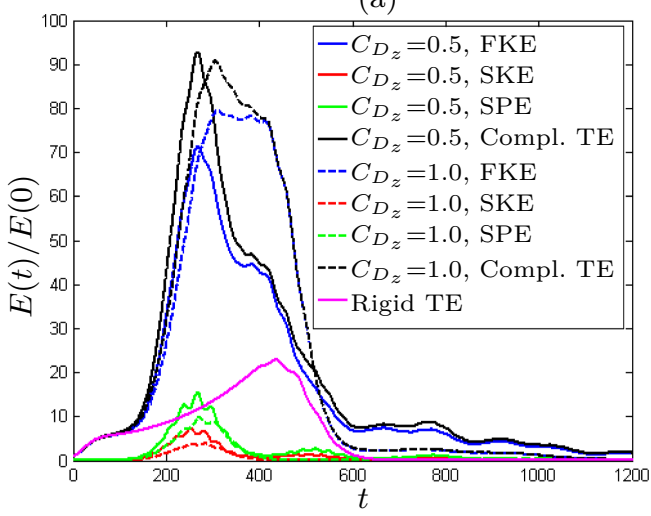

(b)

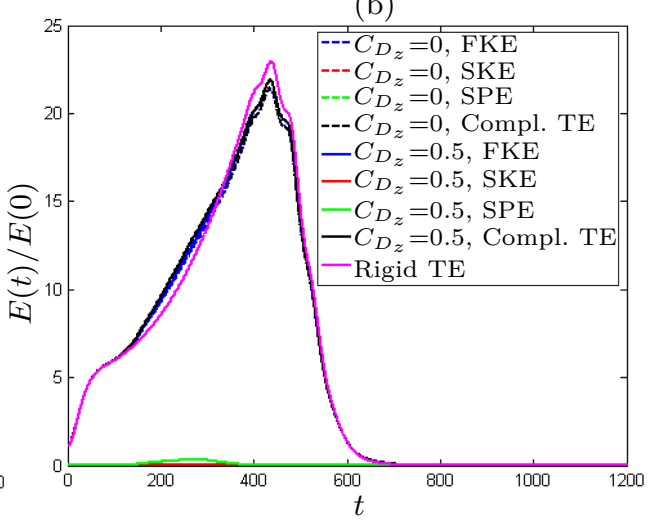

Figure 14: Time evolution of the system total energy (TE) arising from optimal initial disturbances and its decomposition to the flow kinetic (FKE), structural kinetic (SKE) and structural strain (SPE) energy for (a) wall-1 and (b) wall-3. The rigid-wall total (flow-kinetic) energy (TE) is also included.

for structural damping, generates a level of transient growth that is marginally lower than that of flow over a rigid wall; this is seen in figure 14(b). It is also seen that the wall-energy components of the energy growth are almost negligible and that structural damping creates a small increase to the maximum flow kinetic energy (FKE) transfer.

Finally we note that in figure 14(a) the time at which maximum flow kinetic energy (FKE) occurs coincides with the time of the maximum of the wall energies (SPE and SKE) which is earlier than that of the rigid-wall case. We will see below that this difference reflects the arrival of the disturbance at the leading edge and the initiation of compliantwall effects. In contrast, when wall-3 properties are used to generate figure 14(b), there is a time difference between the maxima of the wall energies and the maximum flow kinetic energy that in this case is closely aligned with that of the rigid wall. This is because the much higher stiffness of this wall limits compliant-wall interaction in the transient-growth process.

The different transient dynamics of the two FSI systems as evidenced by the time difference between the maxima of the flow kinetic energy (FKE) and the wall energies (SKE+SPE) are further elucidated by plotting the spatio-temporal evolution of the streamwise disturbance velocity of the wave packet that arises from the optimal initial disturbance. Figures 15(a) and (b) respectively show these for wall-1 with $C_{D_{z}}=0.5$ and wall-3 properties without structural damping. In both cases the optimal initial disturbance is tilted against the shear as is the case for flow over a rigid wall ( $\AA$ kervik et al. 2007). It is then seen to rise to an upright position while extracting energy from the mean flow. Thereafter the wave-packet evolutions in figures 15(a) and (b) differ significantly. In the former, for wall-1 data, the convectively unstable TWF and its interaction with structural modes causes the maximum streamwise velocity to appear above and close to the trailing edge of the compliant panel. When the wave-packet leaves the compliant panel it starts to dissipate since the TWF instability does not exist on the rigidwall section. However, a significant part of the energy accumulated over the compliant panel is distributed to TSWs, which carry the transient-growth energy downstream of the compliant panel. It is remarked that while the FSI system is globally stable, the TWF mode continues to be convectively unstable with a larger spatial amplification rate $\left(\alpha_{i}=-2.13 \times 10^{-2}\right)$ than the least stable TSW mode over the compliant panel 
(a)
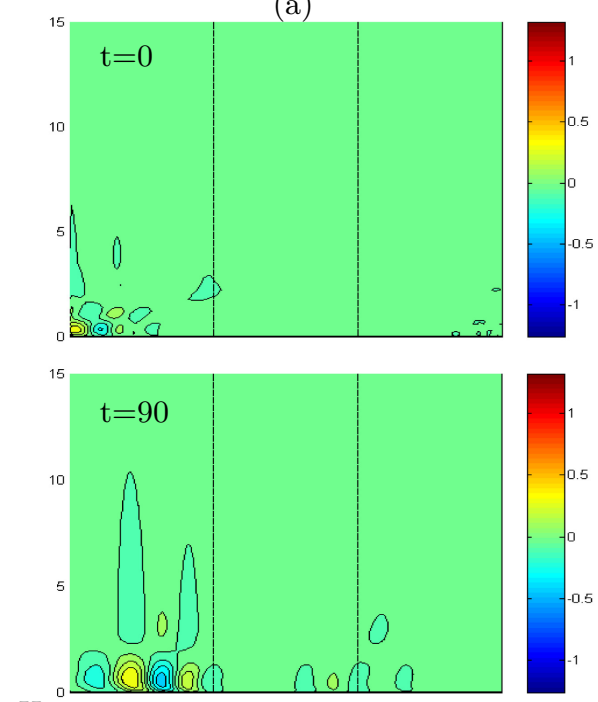

$H$
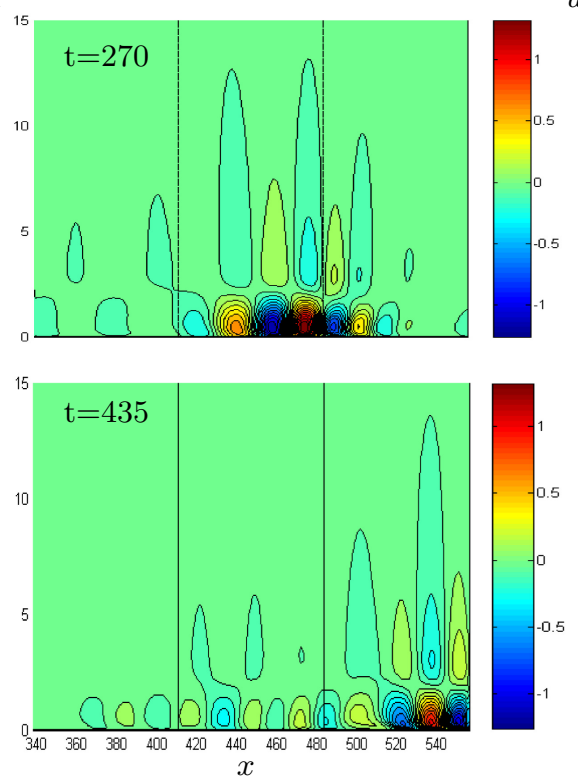

(b)
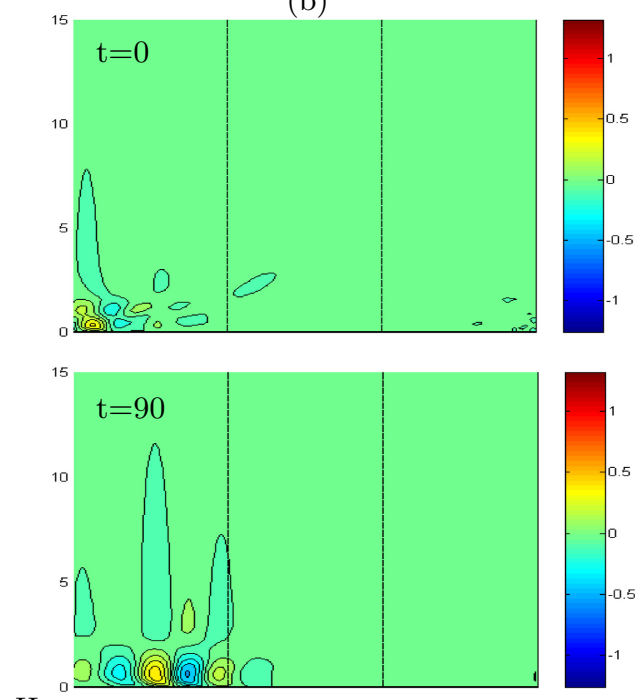

$u_{x} \quad H$
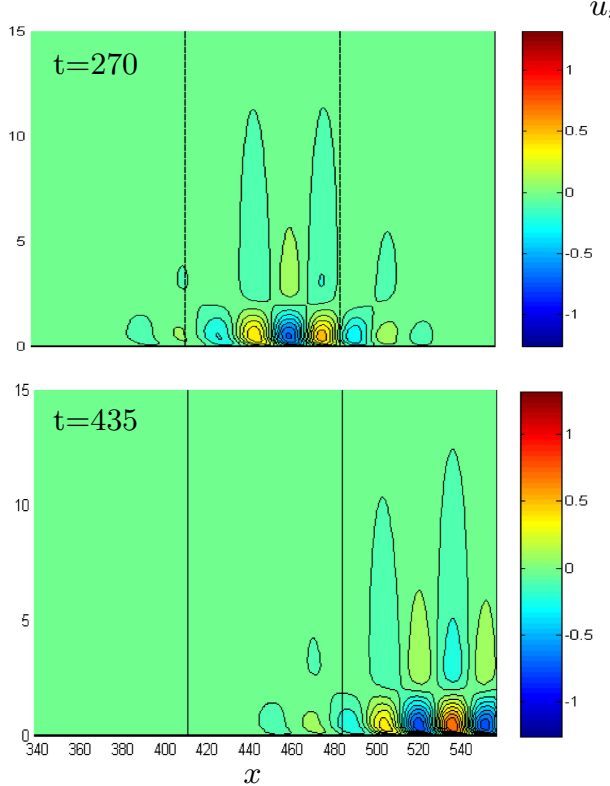

Figure 15: Spatio-temporal evolution of the wave packet of the streamwise disturbance velocity amplitude for (a) wall-1 with $C_{D_{z}}=0.5$ and (b) wall-3 without structural damping. The vertical lines signify the $x$-coordinates of the leading and trailing edges of the compliant panel.

$\left(\alpha_{i}=-8.777 \times 10^{-3}\right)$ but also over the rigid wall $\left(\alpha_{i}=-1.62 \times 10^{-2}\right)$. By contrast, figure 15(b) that pertains to the stiffer wall-3 properties, shows that the maximum amplification of the velocity disturbance is located at the end of the fluid domain. This is because the compliant wall has marginally reduced the transient amplification of the TSW relative to the flow over a rigid wall and for this type of compliant panel the disturbance total energy almost exclusively comprises fluid kinetic energy as seen in figure 14(b). 


\section{Conclusions}

We have formulated a fluid-structure-interaction (FSI) model for Blasius boundarylayer flow fully coupled with the dynamics of a compliant panel with fixed leading and trailing edges embedded in an otherwise rigid wall accounting for both vertical and axial structural displacements. The resulting spatio-temporal analysis is permitted by the hybrid of computational and theoretical modelling used in our novel approach. While we have studied viscous developing flow in the absence of a pressure gradient, our methods could equally be used for the stability analysis of boundary-layer flow developing in a non-zero pressure gradient.

It has been shown that global instability of the linear FSI system can occur through two distinct mechanisms namely, (i) in the wall-based travelling-wave flutter (TWF) eigenvalue branch when its modes interact with a vertical structural mode, and (ii) in the fluid-based Tollmien-Schlichting Wave (TSW) eigenvalue branch when its modes interact with a vertical structural mode. The first features higher temporal growth rates than the second and is insensitive to the perturbation boundary conditions applied to the flow domain. It comprises travelling-wave disturbances of the wall that have characteristic wavelengths far shorter than the panel length and can be suppressed by stiffening the compliant wall. The second is dependent upon the length of the finite panel and the boundary conditions, evidencing a resonant-type behavior with discrete structural modes of the compliant panel. Finally, a globally unstable divergence mode was found at very low oscillatory frequencies that has increasing amplification rate as the free-stream flow speed is increased. The flow speed of its onset is largely unaffected by structural damping. Like the TWF mode, this wall-based instability is insensitive to the boundary conditions applied upstream of the flexible panel. Even though the mechanism for divergence is mainly inviscid in nature, it appears in the present boundary-layer flow only as long as its wavelength is much longer than that of the least stable TSW wave, an effect that has been seen in previous studies (Davies \& Carpenter 1997a).

With the exception of divergence, these types of global instability have not been found before in stability studies of this FSI system. Most studies of the system have used a local stability analysis that assumes, a priori, that TWF and TSWs over a compliant wall are convective instabilities and necessarily ignore the effects of finite panel length. Nevertheless we have shown how local-stability analyses can give insights as to which modes might combine to generate global instability of a finite-length compliant panel. It might have been expected that these temporal instabilities would appear in the numerical simulations of Davies \& Carpenter (1997b) for the analogous system of Poiseuille flow over a compliant insert. However, the TSW-branch phenomenon was not seen because the global instability has a very low growth rate and the numerical simulations were not run for long enough for it to become apparent (Davies 2013). That the TWF-branch of global instability did not appear may be due to the forcing frequency (as the entry condition to the numerical domain) being too low in Davies \& Carpenter (1997b) given that it was chosen to illustrate the development of TSWs over finite compliant panels. The advantage, over numerical simulation, of the modelling developed in the present paper, is that it readily permits investigation and assessment of the full frequency spectrum of system modes. However, for a complete quantification of the globally unstable TSWbranch phenomena, the present methods would need deployed for the full range of inletoutlet boundary conditions.

Both types of global instability can be suppressed by the use of structural damping but would leave the TWF and TSW modes as convectively unstable for the compliant panel properties used herein. It was also shown that the effect of structural damping is 
stabilising for convective TWF instability and destabilising for TSWs in agreement with the findings of previous local-stability analyses and the well-known energy classification of these waves. However, even with damping present, the amplification rate of convectively unstable TSWs are lower in the presence of a compliant panel than over a rigid wall.

The amplification rates of globally unstable modes of the TWF branch have been shown to reduce as the Reynolds number is increased for given free-stream flow speed; this occurs because the critical layer moves away from the wall with increased boundarylayer thickness thereby attenuating its effect in the destabilisation mechanism of the TWF. In contrast, amplification of globally unstable modes on the TSW branch do not exhibit a monotonic change because of the distinct resonant character of the temporal instability with its dependence on the compliant-panel length. However, as Reynolds number is increased the order of structural mode that resonates with the TSW increases.

The inclusion of axial-displacement modes, alongside the vertical-displacement modes, of the compliant wall has been studied. It was shown that the axial structural modes do not combine with modes on either the TSW or TWF branches to create global instability. However, their inclusion causes a very slight increase to the growth rates of the global modes that are based principally upon vertical-displacement structural modes of the compliant panel.

The results of the non-modal analysis developed and deployed in this paper suggest that finite compliant panels capable of attenuating TSWs and which are free from global instability of modes on the TWF branch, via the inclusion of the necessary amount of structural damping, generate levels of transient flow kinetic-energy growth that significantly exceed (by a factor of 3 for the compliant panels assessed in this paper) those that would occur for boundary-layer disturbances over a rigid or very stiff compliant wall. Accordingly, in the design of compliant panels for boundary-layer transition postponement, it is essential to ensure that increased transient growth would not lead to by-pass transition.

The authors gratefully acknowledge the support of the Australian Research Council for the present work through the support of Discovery grant DP1096376 as well as fruitful discussions with Dr Christopher Davies during the revision of this paper.

\section{Appendix A}

The local spatial stability analysis is studied by implementing the Chebyshev collocation matrix combined with the companion matrix method (Bridges \& Morris 1984; Danabasoglu \& Biringen 1990) and solving the eigenvalue problem for the complete spectrum. More specifically, applying the decomposition,

$$
\left\{u_{x}, u_{z}, p, \eta_{x}, \eta_{z}\right\}(x, z, t)=\left\{\hat{u}_{x}, \hat{u}_{z}, \hat{p}, \hat{\eta}_{x}, \hat{\eta}_{z}\right\}(z) \exp (\mathrm{i} \alpha x-\mathrm{i} \omega t)
$$

together with the complex conjugate part of the eigen-decomposition to the linearized $x$ and $z$ components of the Navier-Stokes equations, to the continuity equation, to the normal and tangential force balance, equations (2.9), (2.10) and to the kinematic boundary conditions (2.6) and considering that the disturbances decay to zero at infinity, we obtain the Orr-Sommerfeld equation, 


$$
\begin{gathered}
\left(\alpha^{4} \hat{u}_{z}\right)+\mathrm{i} R e_{x} U_{x}\left(\alpha^{3} \hat{u}_{z}\right)-\left(\mathrm{i} \omega R e_{x}+2 \frac{\mathrm{d}^{2}}{\mathrm{~d} z^{2}}\right)\left(\alpha^{2} \hat{u}_{z}\right) \\
+\mathrm{i} R e_{x}\left(\frac{\mathrm{d}^{2} U_{x}}{\mathrm{~d} z^{2}}-U_{x} \frac{\mathrm{d}^{2}}{\mathrm{~d} z^{2}}\right)\left(\alpha \hat{u}_{z}\right)+\left(\mathrm{i} \omega R e_{x} \frac{\mathrm{d}^{2}}{\mathrm{~d} z^{2}}+\frac{\mathrm{d}^{4}}{\mathrm{~d} z^{4}}\right) \hat{u}_{z}=0
\end{gathered}
$$

1066

with the boundary conditions at the wall $z=0$,

$$
\begin{array}{r}
-\left.C_{A} \frac{\mathrm{d} U_{x}}{\mathrm{~d} z}\right|_{z=0}\left(\alpha^{3} \hat{u}_{z}\right)-\left(C_{A} \omega \frac{\mathrm{d}}{\mathrm{d} z}+\frac{\mathrm{i} \omega^{2}}{R e_{x}}\right)\left(\alpha^{2} \hat{u}_{z}\right) \\
+\left.\left(C_{I} \omega^{2}+\mathrm{i} C_{D_{x}} \omega\right) \frac{\mathrm{d} U_{x}}{\mathrm{~d} z}\right|_{z=0}\left(\alpha \hat{u}_{z}\right) \\
+\left(C_{I} \omega^{2}+\mathrm{i} C_{D_{x}} \omega\right) \omega \frac{\mathrm{d} \hat{u}_{z}}{\mathrm{~d} z}-\frac{\mathrm{i} \omega^{2}}{R e_{x}} \frac{\mathrm{d}^{2} \hat{u}_{z}}{\mathrm{~d} z^{2}}=0 \\
+\left(-\mathrm{i} C_{I} \omega^{2}+C_{D_{z}} \omega+\mathrm{i} C_{K}-\frac{3 \omega}{R e_{x}} \frac{\mathrm{d}}{\mathrm{d} z}\right)\left(\alpha^{2} \hat{u}_{z}\right) \\
+\left.\mathrm{i} \omega \frac{2}{\mathrm{~d} U_{x}}\right|_{z=0}\left(\alpha \hat{u}_{z}\right)+\frac{\omega}{R e_{x}} \frac{\mathrm{d}^{3} \hat{u}_{z}}{\mathrm{~d} z^{3}}+\mathrm{i} \omega^{2} \frac{\mathrm{d} \hat{u}_{z}}{\mathrm{~d} z}=0 \\
\quad\left(\alpha^{3} \hat{u}_{z}\right)
\end{array}
$$

1067 and at $z=\infty$,

1066 
1077

surface element, respectively, are

$$
\begin{aligned}
& I_{i j, k l}^{V X}=\frac{\Delta z_{k l}}{2 \pi} \int_{-\Delta x / 2}^{\Delta x / 2}\left[\left(\frac{z_{i j}}{\left(x_{i j}-\zeta\right)^{2}+z_{i j}^{2}}\right) \mathbf{t}_{k l} \cdot \mathbf{t}_{i j}-\left(\frac{x_{i j}-\zeta}{\left(x_{i j}-\zeta\right)^{2}+z_{i j}^{2}}\right) \mathbf{n}_{k l} \cdot \mathbf{t}_{i j}\right] \mathrm{d} \zeta, \\
& I_{i j, k l}^{V Z}=\frac{\Delta z_{k l}}{2 \pi} \int_{-\Delta x / 2}^{\Delta x / 2}\left[\left(\frac{z_{i j}}{\left(x_{i j}-\zeta\right)^{2}+z_{i j}^{2}}\right) \mathbf{t}_{k l} \cdot \mathbf{n}_{i j}-\left(\frac{x_{i j}-\zeta}{\left(x_{i j}-\zeta\right)^{2}+z_{i j}^{2}}\right) \mathbf{n}_{k l} \cdot \mathbf{n}_{i j}\right] \mathrm{d} \zeta, \\
& I_{i j, k l}^{P X}=\frac{1}{2 \pi} \int_{-\Delta x / 2}^{\Delta x / 2}\left[\left(\frac{x_{i j}-\zeta}{\left(x_{i j}-\zeta\right)^{2}+z_{i j}^{2}}\right) \mathbf{t}_{k l} \cdot \mathbf{t}_{i j}+\left(\frac{z_{i j}}{\left(x_{i j}-\zeta\right)^{2}+z_{i j}^{2}}\right) \mathbf{n}_{k l} \cdot \mathbf{t}_{i j}\right] \mathrm{d} \zeta, \\
& I_{i j, k l}^{P Z}=\frac{1}{2 \pi} \int_{-\Delta x / 2}^{\Delta x / 2}\left[\left(\frac{x_{i j}-\zeta}{\left(x_{i j}-\zeta\right)^{2}+z_{i j}^{2}}\right) \mathbf{t}_{k l} \cdot \mathbf{n}_{i j}+\left(\frac{z_{i j}}{\left(x_{i j}-\zeta\right)^{2}+z_{i j}^{2}}\right) \mathbf{n}_{k l} \cdot \mathbf{n}_{i j}\right] \mathrm{d} \zeta,
\end{aligned}
$$

where $\Delta z_{i j}$ is the cell thickness, $\left(x_{i j}, z_{i j}\right)$ are the physical cell coordinates, and $\mathbf{t}$ and $\mathbf{n}$ are the normal and tangential unit vectors of the specific vortex sheet or source sheet and of the control (collocation) points (Houghton \& Carpenter 2003).

1078 1079 1080

\section{REFERENCES}

ALDER, M. 2015 Development and validation of a fluidâĂŞstructure solver for transonic panel flutter. AIAA Journal 53 (12), 3509-3521.

Alder, M. 2016 Nonlinear dynamics of prestressed panels in low supersonic turbulent flow. AIAA Journal 54 (11), 3632-3646.

Ashpis, D. E. \& Reshotko, E. 1990 The vibrating ribbon problem revisited. Journal of Fluid Mechanics 213, 531-547.

Baltensperger, R. \& Trummer, M. R. 2002 Spectral differencing with a twist. SIAM Journal of Scientific Computing 24, 1465-1487.

Benjamin, T. B. 1963 The three-fold classification of unstable disturbances in flexible surfaces bounding inviscid flows. Journal of Fluid Mechanics 16, 436-450.

Bridges, T. J. \& Morris, P. J. 1984 Differential eigenvalue problems in which the parameter appears nonlinearly. Journal of Computational Physics 55 (3), 437-460.

Bushnell, D. M. 1977 Effects of compliant wall motion on turbulent boundary layers. Physics of Fluids 20, S31-S48.

Butler, K. M. \& FARrell, B. F. 1992 Three-dimensional optimal perturbations in viscous shear flow. Physics of Fluids A 4, 1637-1650.

Canuto, C., Hussaini, M. Y., Quarteroni, A. \& Zang, T. A. 1988 Methods in Fluid Dynamics. Springer-Verlag.

Carpenter, P. W. 1990 Status of transition delay using compliant walls. Progress in Astronautics and Aeronautics 123, 79-113.

CARpenter, P. W. 1991 The optimization of multiple-panel compliant walls for delay of laminar-turbulent transition. American Institute of Aeronautics and Astronautics 91 (1772).

Carpenter, P. W. 1993 Optimization of multiple-panel compliant walls for delay of laminarturbulent transition. American Institute of Aeronautics and Astronautics 31 (7), 11871188.

Carpenter, P. W., Davies, C. \& Lucey, A. D. 2001 Does the dolphin have a secret? Current Science 79, 758-765.

Carpenter, P. W. \& GajJar, J. S. B. 1990 A general theory for two- and three-dimensional wall-mode instabilities in boundary layers over isotropic and anisotropic compliant walls. Theoretical and Computational Fluid Dynamics 1 (6), 349-378.

Carpenter, P. W. \& Garrad, A. D. 1985 The hydrodynamics stability of flow over kramer-type compliant surfaces. part 1.tollmien-schlichting instabilities. Journal of Fluid Mechanics 155, 465-510.

Carpenter, P. W. \& Garrad, A. D. 1986 The hydrodynamics stability of flow over kramer-type compliant surfaces. part 2. flow-induced surface instabilities. Journal of Fluid Mechanics 170, 199-232.

Carpenter, P. W. \& Morris, P. J. 1990 The effects of anisotropic wall compliance on boundary-layer stability and transition. Journal of Fluid Mechanics 218, 171-223.

1081 1082 1083 1084 1085 1086 1087 
\& Kulik, V. M. 1997 Turbulent drag reduction using compliant surfaces. Proceedings of the Royal Society A - Mathematical Physical and Engineering Sciences 453 (1965), $2229-2240$.

Chomaz, J. M. 2005 Global instabilities in spatially developing flows: Non-normality and nonlinearity. Annual Review of Fluid Mechanics 37, 357-392.

Coppola, G. \& DE LuCA, L. 2010 Non-modal dynamics before flow-induced instability in fluid-structure interactions. Journal of Sound and Vibration 329 (7), 848-865.

Cossu, C. \& Chomaz, J. 1997 Global measures of local convective instability. Physical Review Letter 77, 4387-4390.

Crighton, D. G. \& Oswell, J. E. 1991 Fluid loading with mean flow. i. response of an elastic plate to localized excitation. Philosophical Transactions of the Royal Society A 335, $557-592$.

Danabasoglu, G. \& Biringen, S. 1990 A chebyshev matrix method for the spatial modes of the orr-sommerfeld equation. International Journal for Numerical Methods in Fluids 11 (7), 1033-1037.

Davies, C. 2013 Private communication with A. D. Lucey.

Davies, C. \& CARPenter, P. W. $1997 a$ Instabilities in a plane channel flow between compliant walls. Journal of Fluid Mechanics 352, 205-243.

Davies, C. \& Carpenter, P. W. $1997 b$ Numerical simulation of the evolution of tollmienschlichting waves over finite compliant panels. Journal of Fluid Mechanics 335, 361-392.

Davies, C. \& CARPenter, P. W. 2001 A novel velocity-vorticity formulation of the navierstokes equations with applications to boundary layer disturbance evolution. Journal of Computational Physics 172, 119-165.

Dixon, A. E., Lucey, A. D. \& Carpenter, P. W. 1994 The optimization of viscoelastic walls for transition delay. American Institute of Aeronautics and Astronautics 32, 256-267.

Dowell, E. H. 1971 Generalized aerodynamic forces on a flexible plate undergoing transient motion in a shear flow with an application to panel flutter. AIAA Journal 9 (5), 834-841.

Dowell, E. H. 1973 Aerodynamic boundary layer effects on flutter and damping of plates. Journal Aircraft 10 (12), 734-738.

Ehrenstein, U. \& Gallaire, F. 2005 On two-dimensional temporal modes in spatially evolving open flows: The flat-plate boundary layer. Journal of Fluid Mechanics 536, 209-218.

Fukagata, K., Kern, S., Chatelain, P., Koumoutsakos, P. \& Kasagi, N. 2008 Evolutionary optimization of an anisotropic compliant surface for turbulent friction drag reduction. Journal of Turbulence 9 (35), 1-17.

GAD-EL-HAK, M. 1998 Compliant coatings: The simpler alternative. Experimental Thermal and Fluid Science 16, 141-156.

Garrad, A. D. \& Carpenter, P. W. 1982 A theoretical investigation of flow-induced instabilities in compliant coatings. Journal of Sound and Vibration 85 (4), 483-500.

Gaster, M. 1988 Is the dolphin a red herring? In Turbulence Management and Relaminarisation (ed. H. W. Liepmann \& R. Narasimha), pp. 285-304. Springer Berlin Heidelberg.

Hashimoto, A., Aoyama, T. \& Nakamura, Y. 2009 Effects of turbulent boundary layer on panel flutter. AIAA Journal 47 (12), 2785-2791.

Houghton, E. L. \& Carpenter, P. W. 2003 Aerodynamics for Engineering Students, 5th edn. Butterworth-Heinemann.

Huerre, P. \& Monkewitz, P. 1985 Absolute and convective instabilities in free shear layers. Journal of Fluid Mechanics 159, 151-168.

Huerre, P. \& Monkewitz, P. 1990 Local and global instabilities in spatially developing flows. Annual Reviews of Fluid Mechanics 22, 473-537.

Joslin, R. D. \& Morris, P. J. 1992 Effect of compliant walls on secondary instabilities in boundary transition. American Institute of Aeronautics and Astronautics 30, 332-339.

Joslin, R. D., Morris, P. J. \& CArpenter, P. W. 1991 The role of threedimensional instabilities in compliant wall boundary-layer transition. American Institute of Aeronautics and Astronautics 29, 1603-1610.

Katz, J. \& Plotkin, A. 1991 Low Speed Aerodynamics: From Wing Theory to Panel Methods. Mc Graw-Hill.

Kempka, S. N., Strickland, J. H., Glass, M. W., Peery, J. S. \& Ingber, M. S. 1995 Velocity boundary conditions for vorticity formulations of the incompressible navier-stokes

1121

1122 
equations. In Forum on Vortex Methods for Engineering Applications, Sponsored by Sandia National Labs. Sandia National Laboratories.

KIM, E. \& CHOI, H. 2014 Space-time characteristics of a compliant wall in a turbulent channel flow. Journal of Fluid Mechanics 756, 30-53.

Kramer, M. O. 1957 Boundary layer stabilization by distributed damping. Journal of the Aeronautical Sciences 24, 459-460.

Kramer, M. O. 1960 Boundary layer stabilization by distributed damping. Journal of the American Society for Naval Engineers 72, 25-34.

Lehoucq, R. B., Sorensen, D. C. \& YAng, C. 1998 ARPACK Users Guide: Solution of LargeScale Eigenvalue Problems with Implicitly Restarted Arnoldi Methods. SIAM, Philadelphia.

LuCEy, A. 1998 The excitation of waves on a flexible panel in a uniform flow. Philosophical Transactions of the Royal Society of London A 356, 2999-3039.

Lucey, A. \& Carpenter, P. 1995 Boundary layer instability over compliant walls: Comparison between theory and experiment. Physics of Fluids 7 (10), 2355-2363.

Lucey, A. \& PeAKe, N. 2003 Wave excitation on flexible walls in the presence of a fluid flow. In Flow Past Highly Compliant Boundaries and in Collapsible Tubes (ed. P. W. Carpenter \& T. J. Pedley), , vol. 72, chap. 6, pp. 119-145. Springer Netherlands.

Lucey, A. D. \& Carpenter, P. W. 1992 A numerical simulation of the interaction of a compliant wall and inviscid flow. Journal of Fluid Mechanics 234, 121-146.

Lucey, A. D. \& CARPenter, P. W. 1993 On the difference between the hydroelastic instability of infinite and very long compliant panels. Journal of Sound and Vibration 163 (1), 176181.

Luhar, M., Sharma, A. S. \& McKeon, B. J. 2015 A framework for studying the effect of compliant surfaces on wall turbulence. Journal of Fluid Mechanics 768, 415-441.

Luhar, M., Sharma, A. S. \& McKeon, B. J. 2016 On the design of optimal compliant walls for turbulence control. Journal of Turbulence 17 (8), 787-806.

Moler, C. B. \& Stewart, G. W. 1973 An algorithm for generalized matrix eigenvalue problems. SIAM Journal on Numerical Analysis 10 (2), 241-256.

OrR, W. M. 1907 The stability or instability of the steady motions of a perfect liquid and of a viscous liquid. part ii: A viscous liquid. Proceedings of the Royal Irish Academy. Section A: Mathematical and Physical Sciences 27, 69-138.

PAVlov, V. V. 2006 Dolphin skin as a natural anisotropic compliant wall. Bioinspiration and biomimetics 1, 31-40.

PEAKe, N. 2004 On the unsteady motion of a long fluid-loaded elastic plate with mean flow. Journal of Fluid Mechanics 507, 335-366.

Pitman, M. W. \& Lucey, A. D. 2009 On the direct determination of the eigenmodes of finite flow-structure systems. Proceedings of the Royal Society A 465, 257-281.

Pitman, M. W. \& LuceY, A. D. 2010 Stability of plane-Poiseuille flow interacting with a finite compliant panel. In 17th Australasian Fluid Mechanics Conference. University of Auckland.

Åkervik, E., Ehrenstein, U., Gallaire, F. \& Henningson, D. S. 2007 Global twodimensional stability measures of the flat plate boundary-layer flow. European Journal of Mechanics - B/Fluids 27 (5), 501-513.

Rempfer, D., Blossey, P., Parsons, L. \& Lumley, J. 2001 Low-dimensional dynamical model of a turbulent boundary layer over a compliant surface: Preliminary results. In Fluid Mechanics and the Environment: Dynamical Approachess, , vol. 566, pp. 267-283. Springer-Berlin Heidelberg.

Schlichting, H. 1979 Boundary Layer Theory, 7th edn. McGraw-Hill.

Schmid, P. J. 2007 Nonmodal stability theory. Annual Review of Fluid Mechanics 39, 129-162.

Sen, P. K. \& Arora, D. S. 1988 On the stability of laminar boundary-layer flow over a flat plate with a compliant surface. Journal of Fluid Mechanics 197, 201-240.

Shankar, V. \& Kumaran, V. 2002 Stability of wall modes in fluid flow past a flexible surface. Physics of Fluids 14 (7), 2324-2338.

Stewart, P. S., Waters, S. L. \& Jensen, O. E. 2009 Local and global instabilities of flow in a flexible-walled channel. European Journal of Mechanics B/Fluids 28, 541-557.

Theofilis, V. 2003 Advances in global linear instability analysis of nonparallel and threedimensional flows. Progress in Aerospace Sciences 39, 249-315. 
Theofilis, V. 2011 Global linear instability. Annual Review of Fluid Mechanics 43, 319-352.

Tsigklifis, K. \& LuCEY, A. D. 2015 Global instabilities and transient growth in Blasius boundary-layer flow over a compliant panel. Sadhana 40, 945-960.

Wiplier, O. \& Ehrenstein, U. 2000 Numerical simulation of linear and nonlinear disturbance evolution in a boundary layer with compliant walls. Journal of Fluids and Structures 14, $157-182$.

Wiplier, O. \& Ehrenstein, U. 2001 On the absolute instability in a boundary-layer flow with compliant coatings. European Journal of Mechanics - B/Fluids 20, 127-144.

Wu, J. C. \& Thompson, J. F. 1973 Numerical solutions of time-dependent incompressible navier-stokes equations using an integro-differential formulation. Computers and Fluids 1, 197-215.

Xu, S., Rempfer, D. \& Lumley, J. 2003 Turbulence over a compliant surface: numerical simulation and analysis. Journal of Fluid Mechanics 478, 11-34.

YEO, K. S. 1988 The stability of boundary-layer flow over single- and multi-layer viscoelastic walls. Journal of Fluid Mechanics 196, 359-408.

YEO, K. S. 1990 The hydrodynamic stability of boundary-layer flow over a class of anisotropic compliant walls. Journal of Fluid Mechanics 220, 125-160.

YEO, K. S. 1992 The three-dimensional stability of boundary-layer flow over compliant walls. Journal of Fluid Mechanics 238, 537-577.

Yeo, K. S. \& Dowling, A. P. 1987 The stability of inviscid flow over passive compliant walls. Journal of Fluid Mechanics 183, 265-292.

Yeo, K. S., Khoo, B. C. \& Chong, W.K. 1994 The linear stability of boundary-layer flow over compliant walls - effects of boundary-layer growth. Journal of Fluid Mechanics 280, 199-225.

Yeo, K. S., Kноо, B. C. \& Zhaо, H. Z. 1996 The absolute instability of boundary layer flow over viscoelastic walls. Theoretical and Computational Fluid dynamics 8, 237-252.

ZENGL, M. \& RIST, U. 2012 Linear-stability investigations for flow-control experiments related to flow over compliant walls. Nature-Inspired Fluid Mechanics. Note on numerical fluid mechanics and multidisciplinary design 119, 223-237.
1261 